\title{
Conidae (Neogastropoda) assemblage from the Middle Miocene of the Făget Basin (Romania) in the collection of the Hungarian Natural History Museum, Budapest
}

\author{
Zoltán KovÁcs ${ }^{1} \&$ Péter BALÁZs ${ }^{2}$ \\ ${ }^{1}$ H-1077 Budapest, Wesselényi utca 52, Hungary.E-mail: kovacs.zoltan@lisztacademy.hu \\ ${ }^{2}$ H-1115 Budapest, Szentpétery utca 3, Hungary.E-mail: mail.balazs.peter@gmail.com
}

\begin{abstract}
Early Badenian (Middle Miocene) Conidae specimens that were collected from three SW Romanian localities (Coşteiu de Sus, Lăpugiu de Sus, Nemeşeşti), and deposited in the collection of the Hungarian Natural History Museum, Budapest, are revisited. 41 species are described and figured. The material shows affinity with assemblages known from the Börzsöny Mts (Hungary), from the Vienna Basin, and from northern Italy. With 112 figures and 1 table.
\end{abstract}

Key words - Badenian, Central Paratethys, Conidae, Coşteiu de Sus, Lăpugiu de Sus, Middle Miocene, Nemeşeşti, Neogastropoda

\section{INTRODUCTION}

The aim of this paper is the revision of the Early Badenian Conus material that was collected from Coşteiu de Sus, Lăpugiu de Sus, and Nemeşeşti (SW Romania) in the late 19th and early 20th centuries, and is deposited in the collection of the Hungarian Natural History Museum, Budapest. Immensely rich fossil materials are known from the region, however, some fossil groups that were recorded in earlier publications require taxonomical revision. The present work can contribute to the documentation of the actual diversity of the family Conidae (see NAwrot et al. 2015).

Coşteiu de Sus (= Kostej or Felsőkastély in Hungarian), Lăpugiu de Sus (= Felső-Lapugy) and Nemeşeşti (= Nemcse or Nemesesty) are well-known Middle Miocene fossiliferous localities (Fig. 1). All three are located south from the Mureş River, in the small Neogene Făget Basin that represents an eastwards extension of the Pannonian Basin. The Early Badenian fauna of the region is typical of the Central Paratethys.

Tertiary deposits of Lăpugiu with microfossils were first described by Neugeboren $(1846,1847)$. The great geological and palaeontological importance of the site was soon recognized, so numerous papers dealt with the rich fau- 
na and especially with the mollusc assemblages. Conus materials were described by Neugeboren (1853), Stur (1863), Hauer \& Stache (1863), Halaváts (1876, 1881), Hoernes (1878), Hoernes \& Auinger (1879), Boettger (1887), and KocH $(1889,1898)$. The detailed summary of the 19th century achievements was rendered by Косн (1900). Molluscs of Nemeşeşti were first listed by NeUGEBOREN (1852), delineated by HörNEs (1853), and briefly dealt with by Lóczy (1882). The fauna of Coşteiu was described by NeUgeboren (1854), and later analysed by Hoernes \& AUinger (1879), Boettger (1897, 1902-1907), and ZILCH (1934). New researches concerning gastropod faunas from Coşteiu, Lăpugiu or Nemeşeşti were presented by Nițulescu (1931), Moisescu (1955), Duşa (1967, 1969), Papp (1976), Petrescu et al. (1990), PaCAud (2003), CAZe et al. (2010), Tămaş et al. (2013), and Popa et al. (2015). A comprehensive evaluation of the Badenian Conidae assemblage of Lăpugiu was presented by CHIRA \& VoIA (2001).

The taxonomy of the conoids has been widely discussed in the literature (see BOUCHET et al. 2011, KoHN 2014, HENDRICKs 2015). In this paper the genus level classification of Miocene species is based on works of TUCKER \& TENORIO (2009) and LANDAU et al. (2013). Seven genera are applied for the studied material: Kalloconus da Motta, Lautoconus Monterosato, Monteiroconus da Motta, Plagioconus Tucker et Tenorio, Pseudonoduloconus Tucker et Tenorio, Varioconus da Motta, and Conilithes Swainson. For species level revision we used papers of

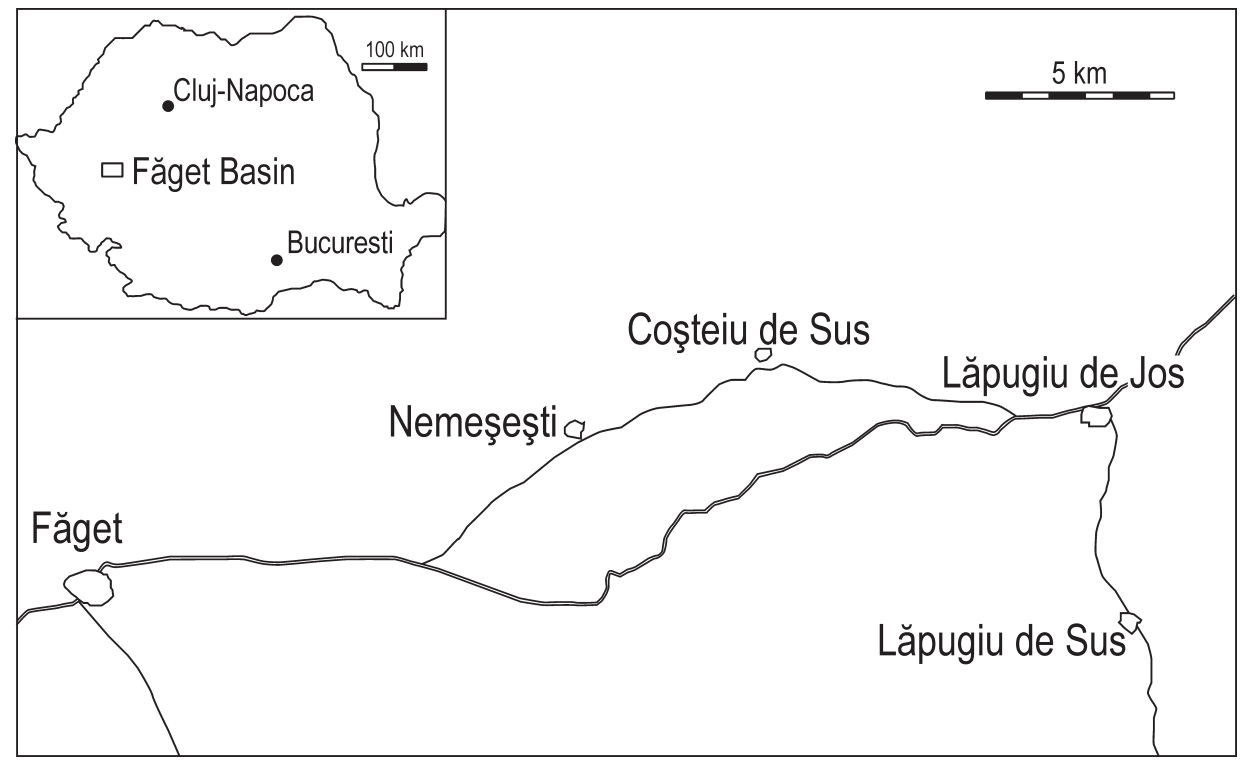

Fig. 1. The studied localities in SW Romania 
Hall (1964), Davoli (1972, 2003), BaŁuK (1997, 2006), Chirli (1997), MuÑIZ (1999), Vaessen (2010), LANdau et al. (2013), Kovács \& Vicián (2014), and JANSSEN et al. $(2014 a, b)$. The following morphological features were taken into consideration: shell size, proportion and overall shape, characteristics of the spire and spiral whorls, types of the shoulder, sculptural features, colour pattern, and subsutural flexure.

\title{
SYSTEMATIC PALAEONTOLOGY
}

As most taxa described here have been discussed in detail in the literature, only selected synonymies are cited which contain the types, the most important synonyms, and the latest papers. Only the Middle Miocene distributions of species are recorded in this paper (for detailed stratigraphic and geographic range see e.g. LANDAU et al. 2013 and Kovács \& VICIÁN 2014), the palaeogeographic scheme is based on that of LANDAU et al. (1. c.). The shell length (SL) is given in $\mathrm{mm}$. All of the photos were taken by P. Balázs.

\author{
Superfamily Conoidea Fleming, 1822 \\ Family Conidae Fleming, 1822 \\ Genus Kalloconus da Motta, 1991
}

Kalloconus berghausi (Michelotti, 1847)

(Figs 2-3, 10-11)

1847 Conus Berghausi - Michelotti, p. 342, pl. 13, fig. 9.

1879 Conus (Dendroconus) Vaceki nov. form. - HoERnEs \& AUINGER, p. 22.

1879 Conus (Dendroconus) Voeslauensis nov. form. - Hoernes \& AUINGER, p. 21, pl. 1, fig. 8, pl. 3, fig. 4.

2013 Kalloconus berghausi (Michelotti) - LANDAU et al., p. 236, pl. 37, figs 6-8, pl. 42, fig. 1, pl. 81, fig. 1 (cum syn.).

Material - 60 specimens - Coşteiu: M 60.8923., 60.8935., 60.8954., 60.8960., 60.9872., 60.9874. (30 mm), 60.9878.A., 60.9911.; Lăpugiu: M 60.7443.A., 60.8289.A-C., 60.8316.A-B., 60.8321.A-E., 60.8334.A-C., 60.8335.A-I., 60.8337.A., 60.8338.A., 60.8339.A-D., 60.8345.R-T., 60.8347.A., 60.8348.A-B., 60.8361.AB., 60.8427.A-B., 60.8919.A-D., 60.8956., 60.8968.A-C., 60.8969.A., 60.8971., 60.9852.A., 60.10134., 60.10135., 60.10203., 62.5998.

Description - SL: 18-77.5. Spire low, outline concave to convex. Spiral whorls smooth. Shoulder rounded. Body whorl broadly conical, outline convex, smooth with fine ridges at the base. Subsutural flexure asymmetrically curved. Colour pattern: spiral rows of small dots. 
Remarks - The taxon was recently revised by LANDAU et al. (2013). By the help of colour pattern analysis, the previous wide species concepts (see e.g. HALL 1964, BA£UK 1997) were rejected, as well as taxonomic validity of some species was verified again (see below).

Distribution - Middle Miocene: Atlantic (France), Paratethys (Austria, Bosnia, Bulgaria, Hungary, Moldavia, Poland, Romania, Serbia, Slovakia, Slovenia, Ukraine), Mediterranean (Italy, Turkey).

\section{Kalloconus daciae (Hoernes et Auinger, 1879)}

(Figs 4-5, 16-18)

1879 Conus (Dendroconus) Daciae nov. form. - Hoernes \& Auinger, p. 21, pl. 3, fig. 1.

2013 Monteiroconus daciae (Hoernes et Auinger) - LANDAU et al., p. 242, pl. 38, fig. 7, pl. 39, fig. 1, pl. 41, fig. 11, pl. 42, fig. 5, pl. 78, fig. 8, pl. 81, fig. 6 (cum syn.).

2014 Lithoconus planospira (Erünal-Erentöz) - KovÁcs \& Vicí́n, p. 75, fig. 41 (cum syn.).

Material - 7 specimens - Coşteiu: M 60.10107., 60.10170.; Lăpugiu: M 60.8275., 60.8278.A., 60.8357.A-B., 62.6019.

Description - SL: 30-80. Spire low to flattened, apex pointed. Spiral whorls slightly concave, striate. Shoulder rounded. Body whorl conical, outline straight, smooth with fine ridges at the base. Subsutural flexure asymmetrically curved.

Remarks - The validity of the taxon was recently dealt with by LANDAU et al. (2013), as well as Conus (Lithoconus) planospira (Erünal-Erentöz, 1958) was regarded as a synonym of $K$. daciae. The species differs from $K$. berghausi in striate spiral whorls. Both $K$. daciae and $K$. hungaricus that were earlier interpreted as synonyms of $K$. berghausi by KovÁcs \& VICIÁN (2014) occurred in the Letkés assemblage (Börzsöny Mts, N Hungary).

Distribution - Middle Miocene: Atlantic (France), North Sea (Belgium), Paratethys (Austria, Bulgaria, Czech Republic, Hungary, Romania, ?Slovakia), Mediterranean (Greece, Italy, Turkey).

Kalloconus fuscocingulatus (Hörnes, 1856)

(Figs 6-7)

1856 Conus fusco-cingulatus Bronn - Hörnes, p. 21, pl. 1, fig. 5.

2014 Dendroconus fuscocingulatus (Bronn in Hörnes) - Kovács \& Vicián, p. 66, figs 43-46.

Material - 25 specimens - Coşteiu: M 60.9862.A-B., 60.10922.; Lăpugiu: M 59.2002., 60.7443.B., 60.7675.A., 60.8289.F-G., 60.8290.A-L., 60.8345.K-L., 60.8973.C., 60.10573., 60.10595.A.

Description - SL: 20-31.5. Spire of moderate height, outline concave. Spiral whorls smooth. Shoulder rounded. Body whorl ventricosely conical, outline 


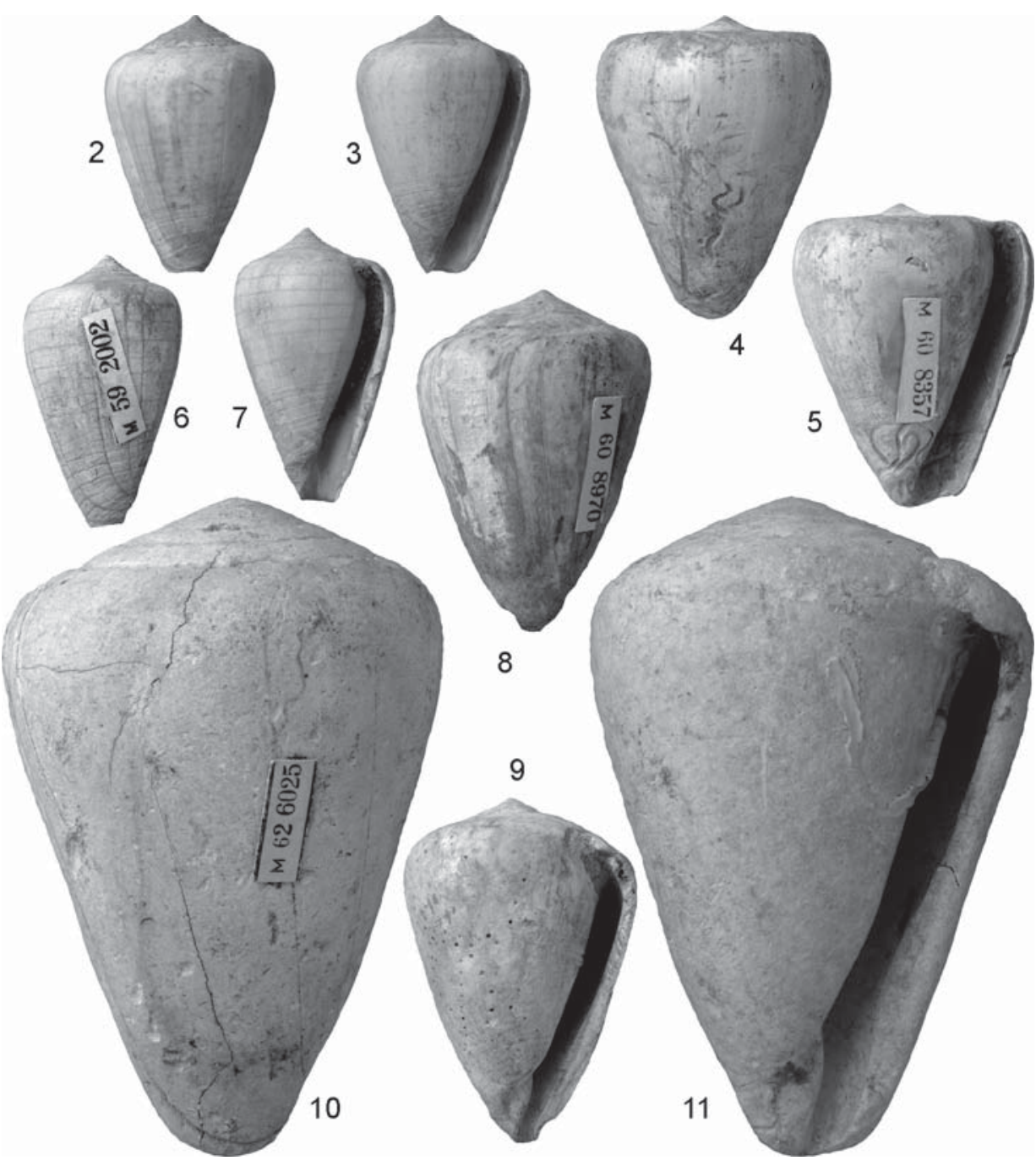

Figs 2-3. Kalloconus berghausi (Michelotti), M 60.9874., Coşteiu, SL: 30, abapertural and apertural views, (1×). - Figs 4-5. Kalloconus daciae (Hoernes et Auinger), M 60.8357.A., Lăpugiu, SL: 35, abapertural and apertural views, (1×). - Figs 6-7. Kalloconus fuscocingulatus (Hörnes), M 59.2002., Lăpugiu, SL: 31.5, abapertural and apertural views, (1×). - Figs 8-9. Kalloconus hungaricus (Hoernes et Auinger), M 60.8970., Lăpugiu, SL: 40, abapertural and apertural views, (1×). - Figs 10-11. Kalloconus berghausi (Michelotti), M 62.5998., Lăpugiu, SL: 77.5, abapertural and apertural views, $(1 \times)$ 
convex, smooth with dense spiral grooves at the anterior end. Subsutural flexure asymmetrically curved. Colour pattern: fine, widely spaced spiral lines.

Remarks - The species shows slight variability of the shell-width. It differs from $K$. berghausi in narrower body whorl, while from the similar Varioconus steinabrunnensis in narrower spire and in rounded shoulder.

Distribution - Middle Miocene: Paratethys (Austria, Bosnia, Bulgaria, Croatia, Czech Republic, Hungary, Poland, Romania, Serbia, Slovakia), Mediterranean (Greece, Italy, Turkey).

\section{Kalloconus hungaricus (Hoernes et Auinger, 1879)}

(Figs 8-9, 19)

1879 Conus (Lithoconus) Hungaricus nov. form. - Hoernes \& AUINGER, p. 29, pl. 2, fig. 6, pl. 4, fig. 1.

2013 Kalloconus hungaricus (Hoernes et Auinger) - LANDAU et al., p. 238, pl. 37, figs 9-10, pl. 38, fig. 1, pl. 41, fig. 8, pl. 42, fig. 2, pl. 81, fig. 2 (cum syn.).

Material - 8 specimens - Coşteiu: M 68.20.; Lăpugiu: M 60.8289.D-E., 60.8341., 60.8361.C., 60.8920., 60.8970., 60.10199.

Description - SL: 26-69. Spire flat to low, outline straight. Spiral whorls flat, striate. Shoulder rounded. Body whorl broadly and ventricosely conical, outline slightly convex, smooth with grooves at the base. Subsutural flexure asymmetrically curved, shallow.

Remarks - The validity of the species was recently treated in detail by LANDAU et al. (2013). K. hungaricus differs from $K$. berghausi in striate spiral whorls and in colour pattern.

Distribution - Middle Miocene: Paratethys (Austria, Hungary, Romania), Mediterranean (Turkey).

\section{Kalloconus steindachneri (Hoernes et Auinger, 1879)}

(Figs 12-15)

1879 Conus (Dendroconus) Steindachneri nov. form. (= Hochstetteri in text) - HoERnes \& AUINGER, p. 24, pl. 3, fig. 3 .

2014 Dendroconus steindachneri (Hoernes et Auinger) - Kovács \& ViCı́́N, p. 67, figs 47-49 (cum syn.).

Material - 29 specimens - Coşteiu: M 60.8949., 60.9872., 60.9892., 60.10113., 68.18.; Lăpugiu: M 60.7675.B-D., 60.8278.B., 60.8335.J., 60.8336.A-G., 60.8338.EF., 60.8932.A., 60.8947., 60.8969.B., 60.8973.A., 60.9852.B., 60.10117.A., 60.10150., 60.10200., 64.222.; Nemeşeşti: M 60.8106.A. 
Description - SL: 28-48. Spire of moderate height, outline straight. Spiral whorls smooth. Shoulder broad, rounded. Body whorl ventricosely conical, outline convex, smooth with grooves at the base. Subsutural flexure slightly curved, oblique.

Remarks - In this paper we follow the classification that was proposed by LANDAU et al. (2013) for the berghausi-group, so Dendroconus steindachneri is
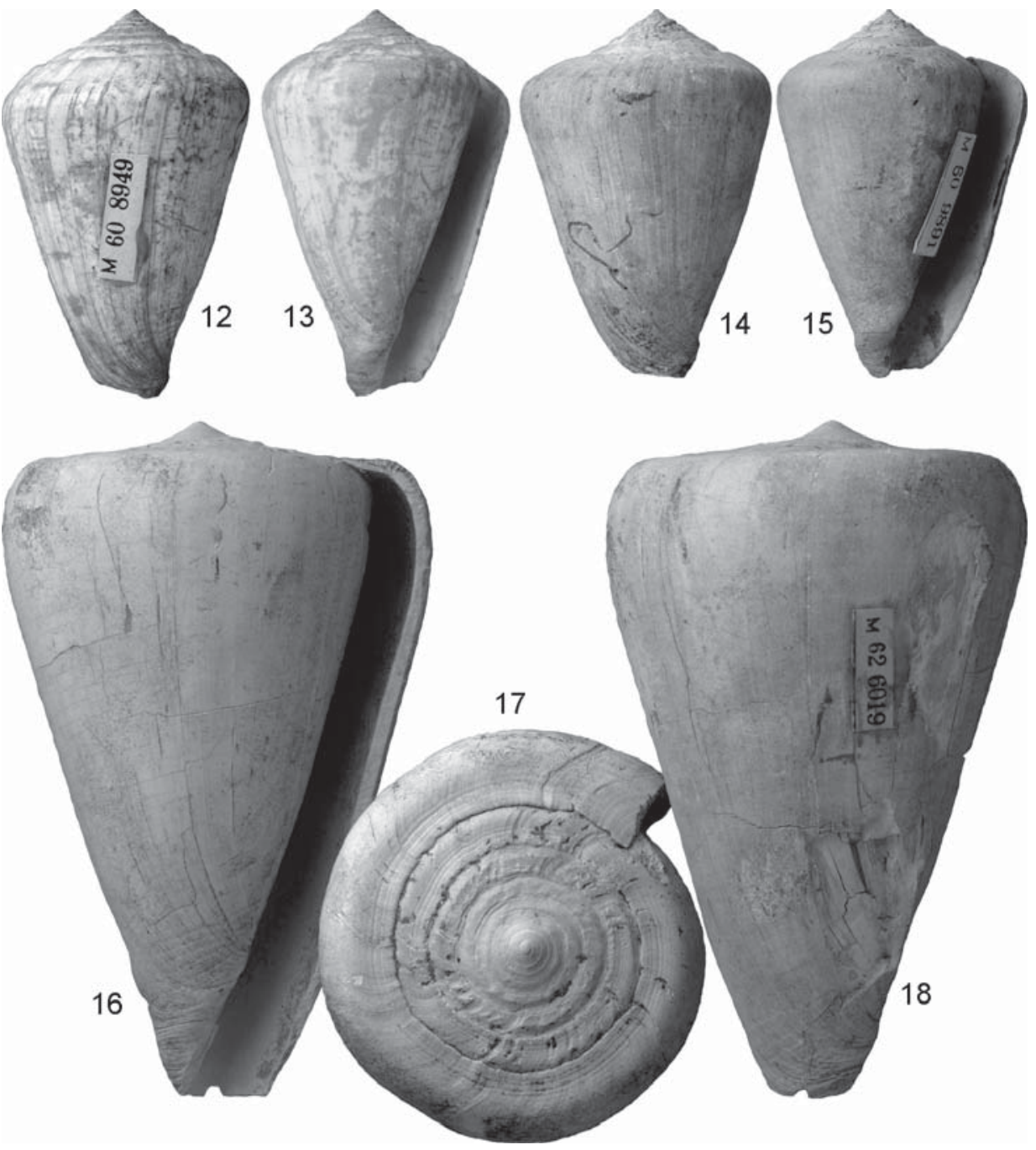

Figs 12-15. Kalloconus steindachneri (Hoernes et Auinger): 12-13. M 60.8949., Coşteiu, SL: 45, abapertural and apertural views, (1×). 14-15. M 60.9892., Coşteiu, SL: 43, abapertural and apertural views, $(1 \times)$. - Figs 16-18. Kalloconus daciae (Hoernes et Auinger), M 62.6019., Lăpugiu, SL: 80 , apertural, apical and abapertural views, $(1 \times)$ 
placed within genus Kalloconus herein. The species shows moderate variability in width of the shoulder. It is closely allied to $K$. berghausi but differs in more elevated and straight spire.

Distribution - Middle Miocene: Paratethys (Austria, Bosnia, ?Croatia, Hungary, Romania, ?Slovenia).

Genus Lautoconus Monterosato, 1923

Lautoconus bitorosus (Fontannes, 1880)

(Figs 20-21)

1880 Conus bitorosus - Fontannes, p. 146, pl. 8, fig. 12.

2013 Lautoconus bitorosus (Fontannes) - LANDAU et al., p. 239, pl. 38, figs 2-4, pl. 41, fig. 9, pl. 42, fig. 3, pl. 81, fig. 3 (cum syn.).

Material - 65 specimens - Coşteiu: M 60.9872., 60.9877.; Lăpugiu: M 59.1858., 60.7675.E., 60.7720., 60.8276.C., 60.8277.A., 60.8289.I., 60.8294.C., 60.8299., 60.8301.A-D., 60.8303., 60.8309.A-B., 60.8313.F-G., 60.8316.C., 60.8318.A., 60.8321.F-J.,60.8337.B.,60.8338.B.,60.8339.F-I.,60.8343.A.,60.8345.I-J.,60.8353.AJ., 60.8360.A-C., 60.8361.D-I., 60.8932.B., 60.8969.C-D., 60.8973.B., 60.9852.C., 60.10132., 60.10184., 60.10311.A., 68.605.A-B.; Nemeşeşti: M 60.8106.B.

Description - SL: 17.4-60. Spire of low to moderate height, outline convex. Spiral whorls smooth. Shoulder subrounded. Body whorl ventricosely conical, outline convex, smooth with fine ridges at the base. Subsutural flexure asymmetrically curved, of moderate depth. Colour pattern: spiral rows of dashes.

Remarks - M. bitorosus varies moderately in height of the spire. It differs from Kalloconus fuscocingulatus in wider spire and in outline of the shoulder. The spire of Varioconus steinabrunnensis and of V. vindobonensis is more concave.

Distribution - Middle Miocene: Atlantic (France, Portugal, Spain), Paratethys (Austria, Hungary, Poland, Romania), Mediterranean (Italy, Tunisia, Turkey).

\section{Lautoconus eschewegi (da Costa, 1866)}

(Figs 22-25)

1866 Conus Eschewegi - DA CosTA, p. 29, pl. 9, figs 18-23.

2014 Lautoconus belus (d’Orbigny) - Kovács \& Vicián, p. 68, figs 4, 53-55.

2014 Lautoconus eschewegi (da Costa) - Kovács \& Vicí́n, p. 70, figs 7, 56-61 (cum syn.).

Material - 13 specimens - Lăpugiu: M 60.7675.F-G., 60.8316.G., 60.8337.G., 60.8339.E., P., 60.8345.M-Q., U-V.; Nemeşeşti: M 60.8118A.

Description - SL: 17-44. Spire narrow, low, outline convex to slightly concave. Spiral whorls smooth, convex. Shoulder rounded. Body whorl ventricosely 


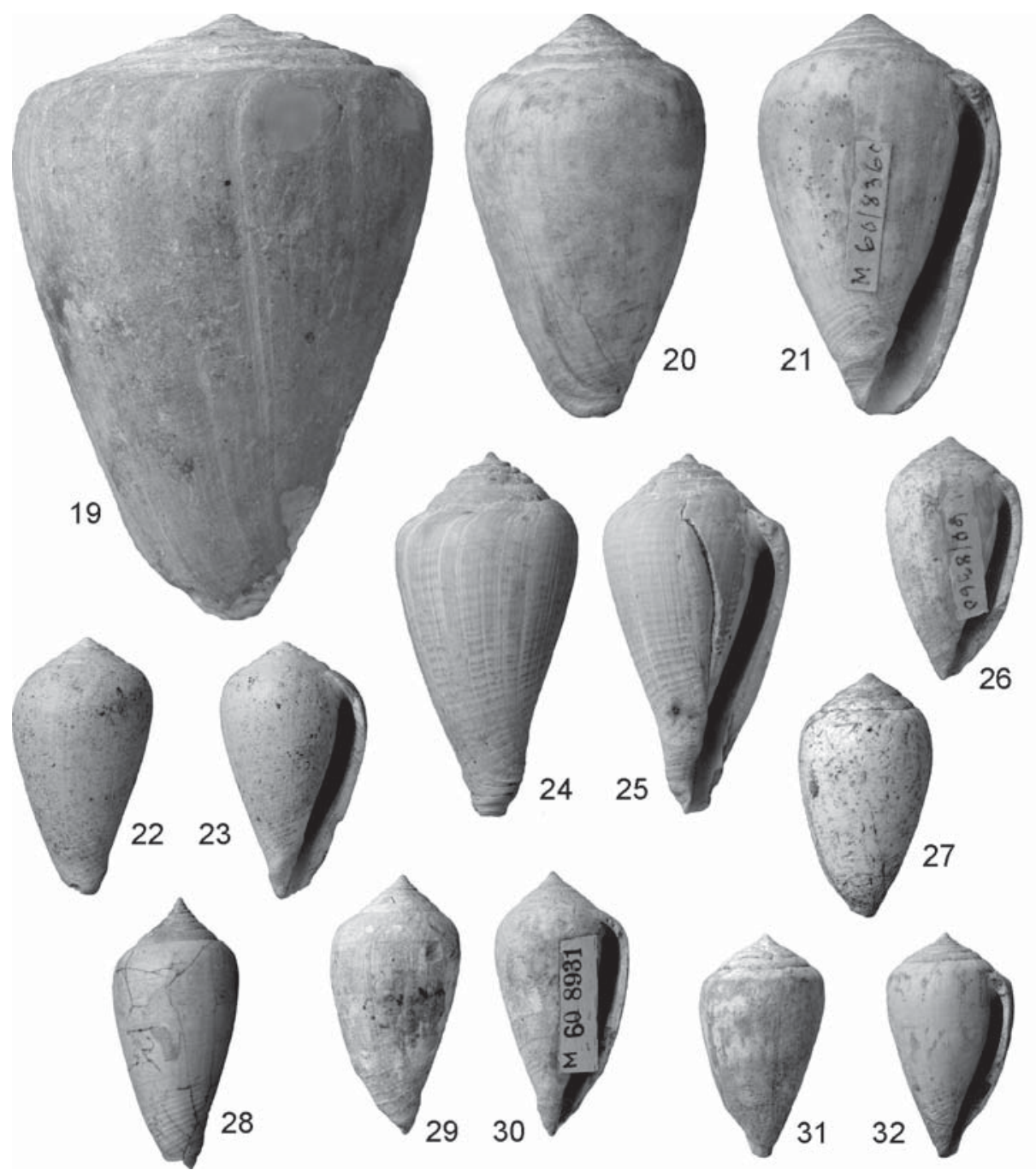

Fig. 19. Kalloconus hungaricus (Hoernes et Auinger), M 60.8341., Lăpugiu, SL: 70, abapertural view, (1×). - Figs 20-21. Lautoconus bitorosus (Fontannes), M 60.8360.A., Lăpugiu, SL: 47, abapertural and apertural views, $(1 \times)$. - Figs 22-25. Lautoconus eschewegi (da Costa): 22-23. M 60.7675.F., Lăpugiu, SL: 29, abapertural and apertural views, (1×). 24-25. M 60.8118.A., Nemeşeşti, SL: 41.5, abapertural and apertural views, $(1 \times)$. - Figs 26-27. Lautoconus miovoeslauensis (Sacco), M 60.8360.D., Lăpugiu, SL: 26, apertural and abapertural views, $(1 \times)$. - Figs 28-30. Lautoconus pyrula (Brocchi): 28. M 60.8948., Lăpugiu, SL: 31, abapertural view, (1×). 29-30. M 60.8931., Lăpugiu, SL: 30, abapertural and apertural views, (1×). - Figs 31-32. Lautoconus rotundus (Hoernes et Auinger), M 60.8291.A., Lăpugiu, SL: 26, abapertural and apertural views, $(1 \times)$ 
conical, outline sigmoid, smooth with fine ridges at the base. Colour pattern consists of spiral rows of thin, widely spaced dashes. Subsutural flexure nearly diagonal.

Remarks - The species shows moderate variability in growth of the spire. Varioconus clavatulus differs in much higher spire, in weakly developed siphonal bend, and in asymmetrically curved subsutural flexure.

Distribution - Middle Miocene: Atlantic (Isles of Azores, Portugal, Spain), Paratethys (Hungary, Romania), Mediterranean (Italy, Morocco, Turkey).

\section{Lautoconus miovoeslauensis (Sacco, 1893)}

(Figs 26-27)

1879 Conus (Chelyconus) ventricosus Bronn - Hoernes \& Auinger, pl. 6, figs 5-6 only.

1893 Conus (Chelyconus) miovoeslauensis - SACCo, p. 108.

2014 Chelyconus miovoeslauensis (Sacco) - Kovács \& Vicián, p. 60, figs 11-15 (cum syn.).

Material - 4 specimens - Lăpugiu: M 60.7443.C., 60.8313.H., 60.8360.D., 60.9032.E.

Description - SL: 22-30. Spire of moderate height, narrow, outline straight to slightly concave. Shoulder rounded. Body whorl ventricosely conical, outline convex, smooth with 4-5 widely spaced spiral grooves at the base. Colour pattern consists of two different types of spiral rows that regularly alternate: a row of small dots and a row of larger dots, visible under UV light. Subsutural flexure asymmetrically curved, of moderate depth.

Remarks - L. miovoeslauensis differs from the closely allied L. bitorosus in oval shell with rounded shoulder and narrower spire, and in colour pattern. In the genus level classification we underline the relationship between the two species. The species was abundant in the Letkés assemblage with two phenotypes (smooth or granulate shells) (Kovács \& VICIÁN 2014). From Lăpugiu only four specimens can be recorded, all bear smooth shell.

Distribution - Middle Miocene: Paratethys (Austria, Hungary, Romania).

\section{Lautoconus pyrula (Brocchi, 1814)}

(Figs 28-30)

1814 Conus pyrula - BRocchi, p. 288, pl. 2, fig. 8.

1879 Conus (Chelyconus) Sturi nov. form. - Hoernes \& AuInger, p. 41, pl. 5, figs 9-10.

2014 Lautoconus pyrula (Brocchi) - Kovács \& ViCián, p. 71, figs 62-65 (cum syn.).

Material - 13 specimens - Coşteiu: M 59.1926.; Lăpugiu: M 59.1847., 60.8287.A-B., 60.8289.H., 60.8316.E-F., 60.8317.C., 60.8345.G-H., 60.8931., 60.8948., 60.10153 . 
Description - SL: 23-37. Spire of moderate height, outline sigmoid. Apex elevated, spiral whorls smooth. Shoulder rounded. Body whorl ventricosely conical, outline slightly sigmoid, smooth with fine incised grooves at the anterior end. Subsutural flexure slightly curved, nearly diagonal.

Distribution - Middle Miocene: Atlantic (Portugal, Spain), Paratethys (Austria, Bosnia, Czech Republic, Hungary, Romania, Serbia), Mediterranean (Greece, Italy, Turkey).

\section{Lautoconus rotundus (Hoernes et Auinger, 1879)}

(Figs 31-32)

1879 Conus (Chelyconus) rotundus nov. form. - Hoernes \& AUINGER, p. 50, pl. 6, fig. 8.

2014 Varioconus rotundus (Hoernes et Auinger) - KovÁcs \& Vicián, p. 61, figs 16-19 (cum syn.).

Material - 5 specimens - Lăpugiu: M 59.2001., 60.8291.A-D.

Description - SL: 15-36. Spire moderately high, outline convex, apex projected. Spiral whorls convex, smooth, suture deep. Shoulder rounded to subangulate. Body whorl broadly and ventricosely conical, outline straight to slightly convex, smooth with fine spiral ridges at the anterior end. Subsutural flexure asymmetrically curved, shallow.

Remarks - The species is closely allied to L. bitorosus in morphology, so it is classified here within genus Lautoconus. L. bitorosus differs in shape of the body whorl and the shoulder. L. rotundus differs from Varioconus conoponderosus in lower and convex spire.

Distribution - Middle Miocene: Paratethys (Austria, Hungary, Poland, Romania).

\section{Lautoconus ventricosus (Gmelin, 1791)}

(Figs 33-34)

1791 Conus ventricosus - GMelin, p. 3397.

2014 Lautoconus ventricosus (Gmelin) - Kovács \& Vicián, p. 73, figs 70-72 (cum syn.).

Material - 6 specimens - Lăpugiu: M 59.2004.B., 60.9852.D., 60.10152., 68.601., 68.603., 68.605.C.

Description - SL: 25-42. Spire of moderate height, outline straight. Spiral whorls smooth. Shoulder angulate. Body whorl ventricosely conical, outline slightly convex, smooth with fine ridges at the base. Subsutural flexure nearly diagonal.

Remarks - The species is characterized by high level of intraspecific variability. 
Distribution - Recent L. ventricosus occurs in the Mediterranean Sea and in the Eastern Atlantic Ocean (Portugal to Senegal). Middle Miocene records: North Sea (Belgium), Paratethys (Austria, Hungary, Romania), Mediterranean (Italy).

Genus Monteiroconus da Motta, 1991

Monteiroconus mercati (Brocchi, 1814)

(Figs 35-36, 40-41)

1814 Conus Mercati - BrocchI, p. 287, pl. 2, fig. 6.

2013 Monteiroconus mercati (Brocchi) - LANDAU et al., p. 244, pl. 39, figs 2-4, pl. 41, fig. 12, pl. 42, fig. 6, pl. 81, figs 7-8 (cum syn.).

Material - 8 specimens - Coşteiu: M 60.9865.A-B., 68.23.; Lăpugiu: M 60.8293.C., 60.8360.J-K., 60.10149., 60.10198.

Description - SL: 50-60. Spire of low to moderate height, outline slightly gradate, straight to slightly concave. Spiral whorls smooth, sutural ramps flat to gently concave. Shoulder angulate. Body whorl conical, slightly elongate, outline convex, smooth with fine ridges at the base. Subsutural flexure asymmetrically curved, of moderate depth.

Remarks - The species shows moderate variability of growth of the spire. The specimen on Figs 40-41 bears gradate spire with subangular and smooth spiral whorls, it agrees with $M$. mercati var. turricula (Brocchi).

Distribution - Middle Miocene: Atlantic (France), Paratethys (Austria, Czech Republic, Hungary, Romania), Mediterranean (Turkey).

\section{Monteiroconus mojsvari (Hoernes et Auinger, 1879)}

(Figs 37-39)

1879 Conus (Dendroconus) Mojsvari nov. form. - Hoernes \& Auinger, p. 18, pl. 3, fig. 2.

1973 Conus (Lithoconus) mercati miocaenicus Sacco - Nicorici \& SAgATovicI, p. 175, pl. 27, fig. 1. ? 2001 Conus (Lithoconus) mercatti [sic] miocenicus Sacco - CHIRA \& Vora, pl. 2, fig. 3.

2013 Monteiroconus mojsvari (Hoernes et Auinger) - LANDAU et al., p. 243 (cum syn.).

Material - 1 specimen - Lăpugiu: M 62.6027.

Description - SL: 88. Spire low, outline slightly concave. Spiral whorls canaliculate, striate. Shoulder subangular. Body whorl conical, outline straight, smooth with fine ridges at the base. Subsutural flexure asymmetrically curved.

Remarks - The validity of the taxon was recently verified by LANDAU et al. (2013). M. mercati differs from M. mojsvari in gradate spire with smooth whorls.

Distribution - Middle Miocene: Paratethys (Austria, Hungary, Italy, Romania). 


\section{Monteiroconus tietzei (Hoernes et Auinger, 1879)}

(Figs 42-43)

1879 Conus (Lithoconus) Tietzei nov. form. - Hoernes \& AUINGer, p. 28, pl. 1, fig. 3.

2014 Monteiroconus tietzei (Hoernes et Auinger) - Kovács \& Vicián, p. 79, figs 92-96 (cum syn.).

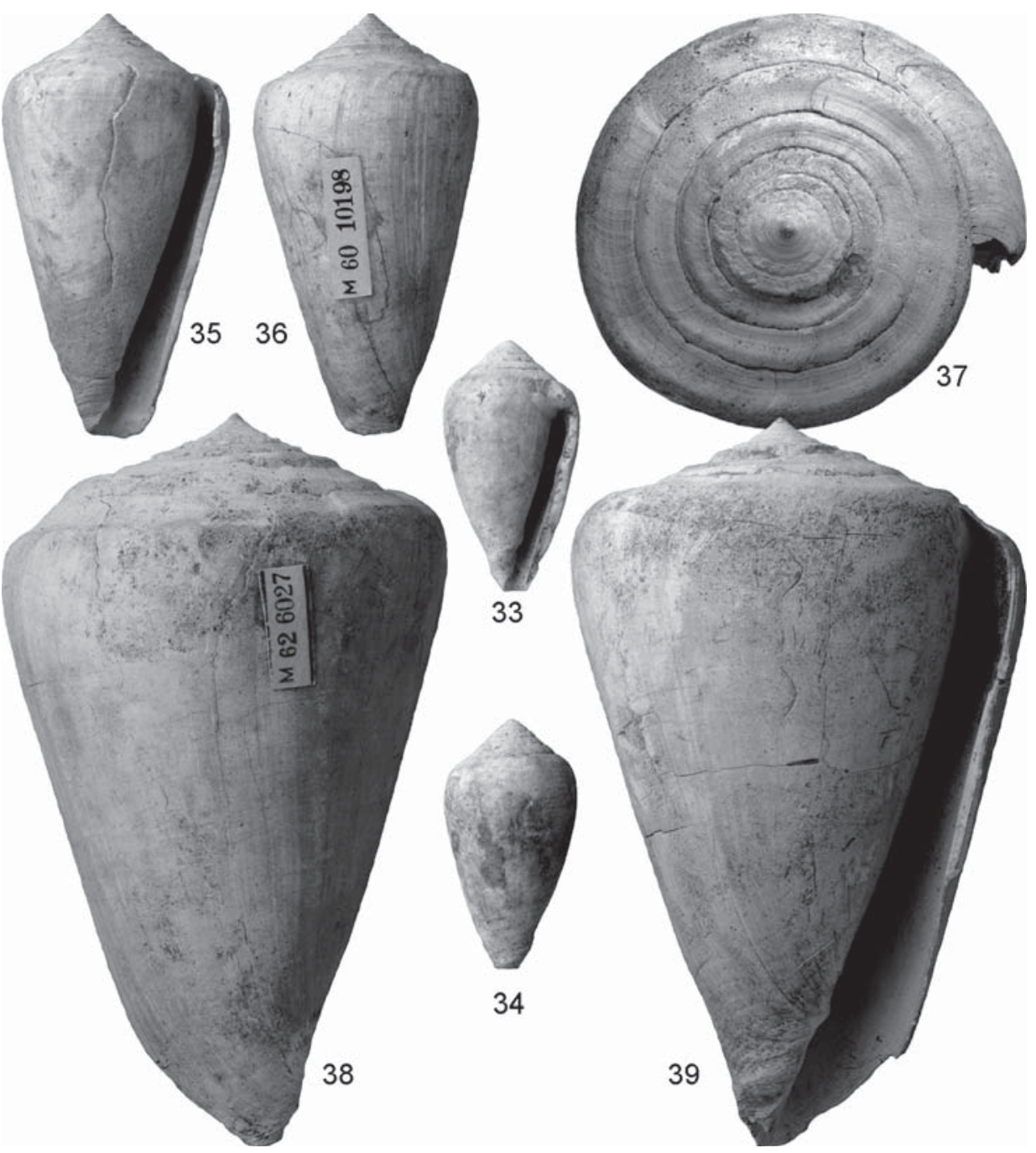

Figs 33-34. Lautoconus ventricosus (Gmelin), M 60.10152., Lăpugiu, SL: 29, apertural and abapertural views, (1×). - Figs 35-36. Monteiroconus mercati (Brocchi), M 60.10198., Lăpugiu, SL: 50, apertural and abapertural views, $(1 \times)$. - Figs 37-39. Monteiroconus mojsvari (Hoernes et Auinger), M 62.6027., Lăpugiu, SL: 88, apical, abapertural and apertural views, $(1 \times)$ 
Material - 12 specimens - Lăpugiu: M 60.8278.C., 60.8293.A., 60.8335.K., 60.8339.J., 60.8346.A., 60.8347.B., 60.8945., 60.8968.D., 60.8972., 60.9852.E., 60.10162.; Nemeşeşti: M 60.8107.

Description - SL:23-38. Spire of low to moderate height, outline concave. Spiral whorls striate. Shoulder subangulate. Body whorl conical, outline straight to slightly convex, smooth with fine ridges at the base. Subsutural flexure asymmetrically curved.

Remarks - The species differs from Kalloconus berghausi in higher and concave spire, and in subangular shoulder. M. mercati differs in elevated spire and in elongate body whorl.

Distribution - Middle Miocene: Paratethys (Bosnia, Hungary, Romania).

Genus Plagioconus Tucker et Tenorio, 2009

Plagioconus elongatus (Borson, 1820)

(Figs 44-45)

1820 Conus Elongatus - Borson, p. 198, pl. 1, fig. 4.

1856 Conus Haueri Partsch - Hörnes, p. 34, pl. 4, fig. 4 only.

1972 Conus elongatus Borson - DAvoli, p. 105, tab. 12, text-fig. 25, pl. 6, figs 18-20, 22-24.

2006 Conus (Leptoconus) elongatus Borson - BA£UK, p. 216, pl. 16, fig. 8.

Material: 1 specimen - Coşteiu: M 60.7860.

Description - SL: 86.5. Spire of moderate height, outline slightly concave. Spiral whorls concave, smooth. Suture deep. Shoulder broad, rounded. Body whorl elongate, outline straight, smooth with fine grooves on the lower third. Subsutural flexure asymmetrically curved, deep.

Remarks $-P$. extensus is a closely allied form, but differs in elevated, narrow, gradate spire with convex, angulate whorls.

Distribution - Middle Miocene: Paratethys (Austria, Bulgaria, Hungary, Poland, Romania).

\section{Plagioconus extensus (Hörnes, 1856)}

(Figs 46-47)

1856 Conus extensus Partsch - Hörnes, p. 37, pl. 5, fig. 1.

non 2001 Conus (Leptoconus) extensus Partsch - CHIRA \& VoiA, pl. 2, fig. 1. [= P. marii (Sacco)] 2014 Plagioconus extensus (Partsch in Hörnes) - Kovács \& Vicián, p. 79, figs 97-100 (cum syn.).

Material - 14 specimens - Coşteiu: M 60.9859., 60.9889., 68.15., 68.21.; Lăpugiu: M 60.8282.A., 60.8286.A-B., 60.8358.G., 60.8359.A-B., 60.8938., 60.10060., 68.594., Nemeşeşti: M 60.8135. 

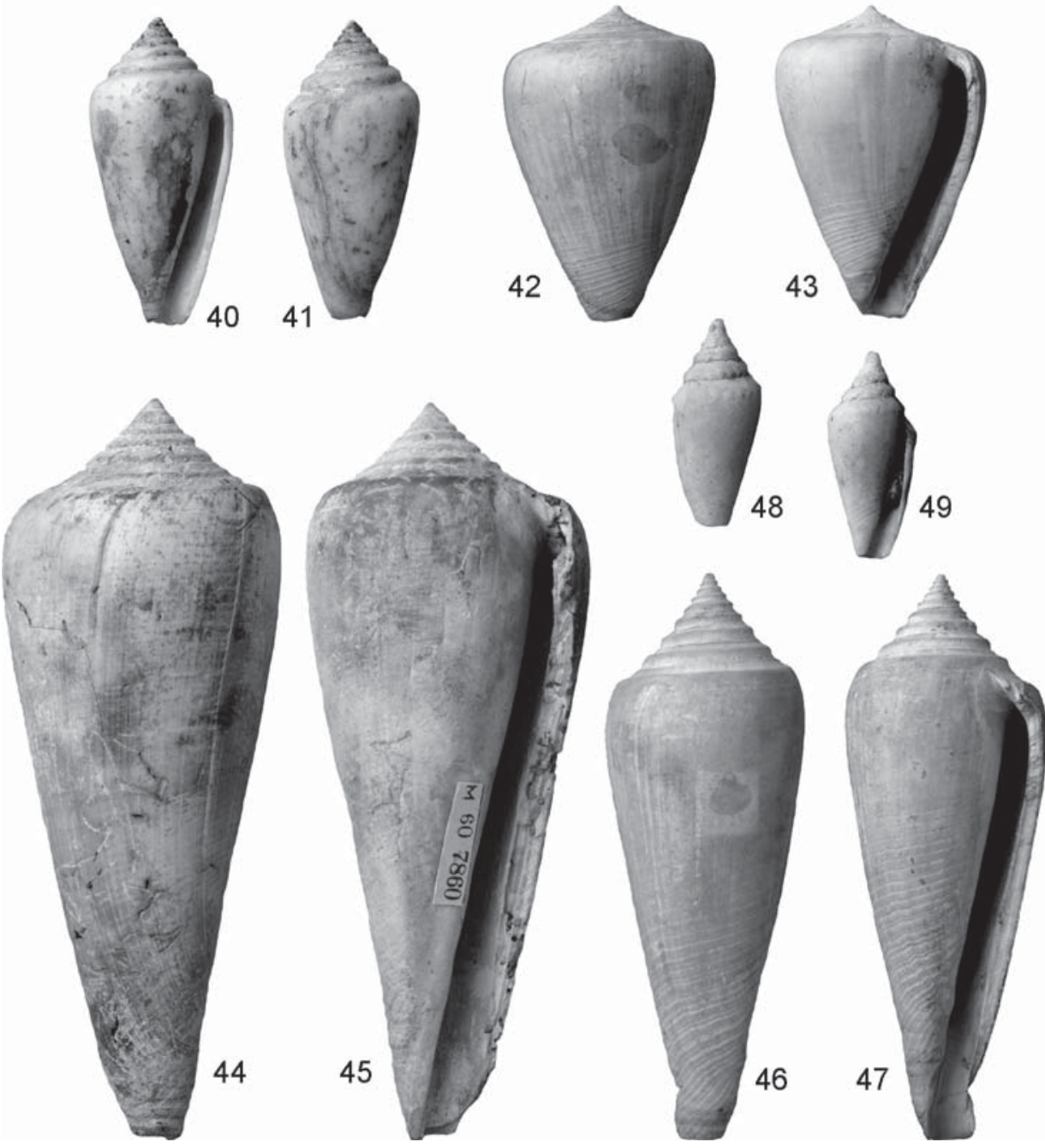

Figs 40-41. Monteiroconus mercati (Brocchi), M 60.9865.A., Coşteiu, SL: 34, apertural and abapertural views, (1×). - Figs 42-43. Monteiroconus tietzei (Hoernes et Auinger), M 60.8107., Nemeşeşti, SL: 36, abapertural and apertural views, $(1 \times)$. - Figs 44-45. Plagioconus elongatus (Borson), M 60.7860., Coşteiu, SL: 86.5, abapertural and apertural views, $(1 \times)$. - Figs 46-47. Plagioconus extensus (Hörnes), M 60.8135., Nemeşeşti, SL: 64, abapertural and apertural views, (1×). - Figs 48-49. Pseudonoduloconus cf. subbigranosus (Sacco), M 60.9873.B., Coşteiu, SL: 8, abapertural and apertural views, $(3 \times)$ 
Description - SL: 37-89. Spire high, outline straight, step-like. Subapical whorls finely tuberculate, late whorls striate. Shoulder rounded. Body whorl narrowly conical, outline straight, smooth with marked, widely spaced grooves on the lower half. Subsutural flexure asymmetrically curved and deep.

Remarks - P. elongatus differs in growth of the spire and in robust shape of the shoulder.

Distribution - Middle Miocene: Paratethys (Austria, Bulgaria, Hungary, Romania).

Plagioconus marii (Sacco, 1893)

(Figs 50-51)

1893 Conus (Chelyconus) Marii - SAcco, p. 62, pl. 6, fig. 1; varieties: fusulopupoides, ovatopupoides, digitiformis, asparagispira, perfusulospira, clavatoidea, subconicospira, medioventrosa, subpileospira, ovatobrevis, pileospira, mamillatospira: p. 62-64, pl. 6, figs 2, 4-8, 10-15. 2014 Plagioconus marii (Sacco) - Kovács \& Vicián, p. 81, figs 101-105, 108 (cum syn.).

Material - 1 specimen - Coşteiu: M 60.10096.

Description - SL: 66. Spire elevated, outline convex. Spiral whorls convex, smooth. Suture deep. Shoulder broad, rounded. Body whorl elongate, ventricosely conical, smooth with ridges at the base. Aperture narrow, straight. Subsutural flexure asymmetrically curved.

Remarks - The species shows high level of intraspecific variability. P. puschi differs in lower and narrower spire.

Distribution - Middle Miocene: Paratethys (Hungary, Romania), Mediterranean (Italy).

\section{Plagioconus puschi (Michelotti, 1847)}

(Figs 52-53)

1847 Conus Puschi - Michelotti, p. 340, pl. 14, fig. 6.

2013 Plagioconus puschi (Michelotti) - LANDAU et al., p. 245, pl. 39, fig. 5, pl. 41, fig. 13, pl. 42, fig. 7, pl. 81, fig. 9 (cum syn.).

Material - 11 specimens - Coşteiu: M 60.9883., 60.9891., 60.9912.; Lăpugiu: M 60.8293.B., 60.8358.H., 60.8359.C., 60.8367.A-B., 68.599.A., 68.604.; Nemeşeşti: M 60.8136.

Description - SL: 40-71. Spire conical, moderately high, outline straight. Spiral whorls convex. Shoulder rounded. Body whorl narrowly conical, outline straight, smooth with fine ridges on the lower third. Subsutural flexure diagonal.

Remarks - The species differs from the closely allied forms (P. elongatus, $P$. extensus, $P$. marii) in growth of the spire, and in subsutural flexure. 
Distribution - Middle Miocene: Atlantic (France, Portugal), Paratethys (Austria, Bosnia, Bulgaria, Hungary, Romania, Serbia), Mediterranean (Greece, Turkey).
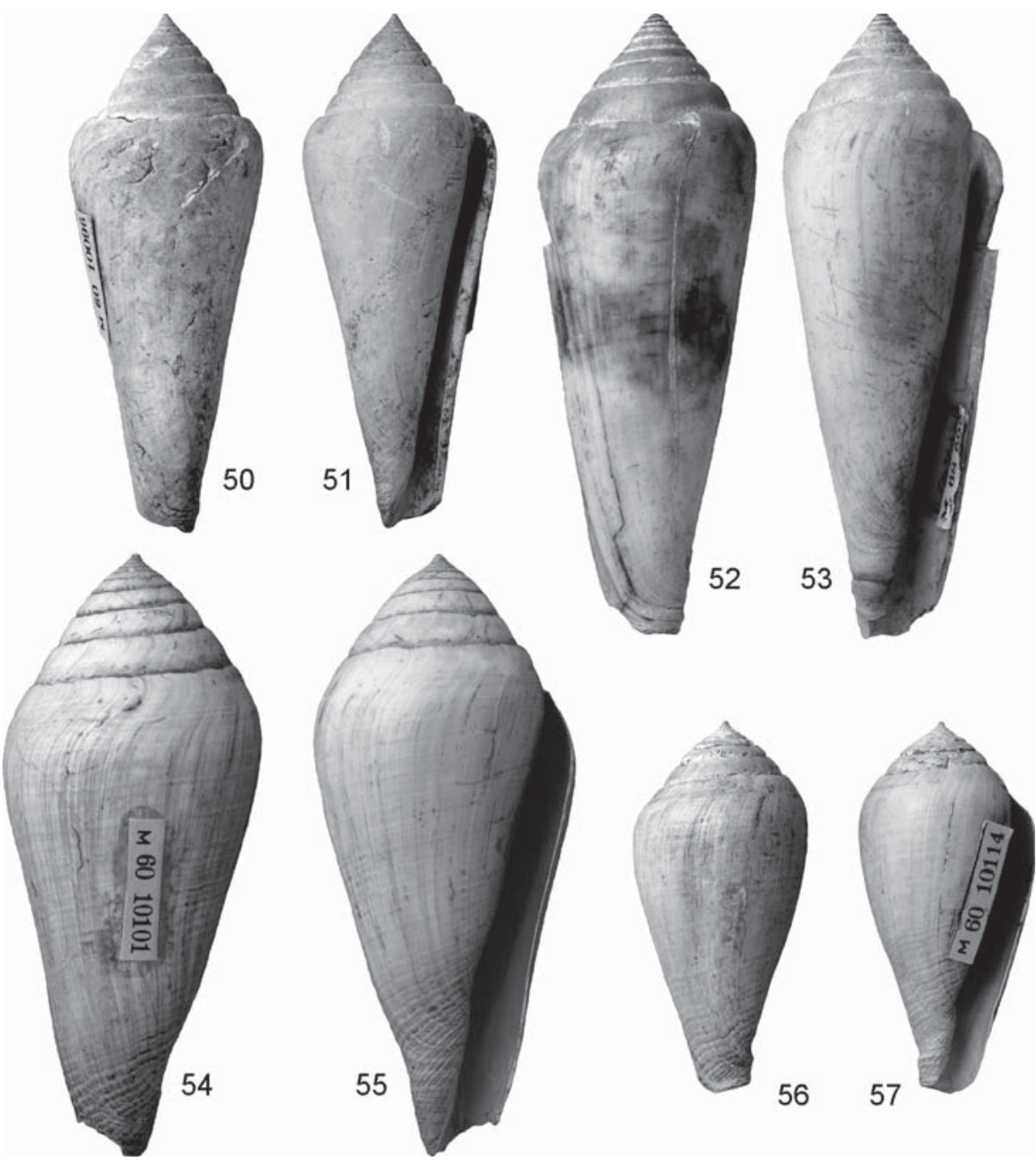

Figs 50-51. Plagioconus marii (Sacco), M 60.10096., Coşteiu, SL: 61, abapertural and apertural views, (1×). - Figs 52-53. Plagioconus puschi (Michelotti), M 68.599.A., Lăpugiu, SL: 73, abapertural and apertural views, $(1 \times)$. - Figs 54-57. Varioconus clavatulus (Orbigny): 54-55. M 60.10101., Coşteiu, SL: 71, abapertural and apertural views, (1×). 56-57. M 60.10114., Coşteiu, SL: 43, abapertural and apertural views, $(1 \times)$ 
Genus Pseudonoduloconus Tucker et Tenorio, 2009

Pseudonoduloconus cf. subbigranosus (Sacco, 1893)

(Figs 48-49)

1893 Stephanoconus subbigranosus - SAcco, p. 120, pl. 11, fig. 24, varieties: bispirata, subbicrenulata, ligusticofusulata, ligusticovulata, ligusticoconica, pliocoronaxoides: p. 120-121, pl. 11, figs $25-30$.

1999 Conus (Stephanoconus) subbigranosus (Sacco) - MUÑIZ, p. 86, fig. 10J-L.

Material - 1 specimen - Coşteiu: M 60.9873.B.

Description - SL: 8. Spire elevated, outline slightly concave. Spiral whorls convex, beaded. Shoulder rounded, beaded. Body whorl conical, outline straight, smooth with spiral ridges at the base.

Remarks - Our specimen bears only 5 spiral whorls, it is regarded as a juvenile form, so the use of open nomenclature seems reasonable. It differs from the type (refigured by FERrero MORTARA et al. 1984, pl. 20, fig. 12) in slightly narrower and higher spire, it is closer to the morphotype subbicrenulata (SACCO 1893, pl. 11, fig. 26). The small-sized species differs from $C$. antidiluvianus and $C$. dujardini in convex spiral whorls and rounded shoulder.

Distribution - Middle Miocene: Paratethys (Romania), Mediterranean (Italy).

Genus Varioconus da Motta, 1991

Varioconus clavatulus (Orbigny, 1852)

(Figs 54-57)

1852 Conus clavatulus - OrBigNY, p. 11.

2014 Varioconus clavatulus (Orbigny) - KovÁcs \& VicıáN, p. 83, figs 109-110 (cum syn.).

Material - 3 specimens - Coşteiu: M 60.10101., 60.10114.; Nemeşeşti: M 60.8118.B.

Description - SL: 43-71. Spire of moderate height, outline convex. Spiral whorls high, slightly convex, smooth. Suture deep. Shoulder rounded. Body whorl sigmoid, smooth with fine ridges at the base. Subsutural flexure asymmetrically curved, of moderate depth.

Remarks - V. noe differs in striate spiral whorls and in narrower body whorl.

Distribution - Middle Miocene: North Sea (Belgium, the Netherlands), Paratethys (Hungary, Romania), Mediterranean (Turkey). 
Varioconus conoponderosus (Sacco, 1893)

(Figs 58-59)

1893 Conus (Chelyconus) conoponderosus - SACco, p. 75, pl. 7, fig. 22, varieties: conicissima, subpupoidea: p. 75-76, pl. 7, figs 23-24.

2014 Varioconus conoponderosus (Sacco) - Kovács \& Vicián, p. 83, figs 111-115 (cum syn.).

Material - 9 specimens - Coşteiu: M 60.8952.; Lăpugiu: M 60.8290.M., 60.8311., 60.8312.A., 60.8337.C., 60.8338.C-D., 60.8930., 68.598.

Description - SL: 31-44. Spire of low to moderate height, outline slightly convex. Spiral whorls smooth. Shoulder rounded to subangulate. Body whorl conical, outline straight to slightly convex, smooth with pronounced growth lines, and with fine grooves at the base. Subsutural flexure asymmetrically curved.

Remarks - The species is characterized by intraspecific variability (DAVOLI 1972). V. ponderosus differs in more elevated spire.

Distribution - Middle Miocene: Paratethys (?Austria, Hungary, Romania), Mediterranean (?Italy, Turkey).

Varioconus montisclavus (Sacco, 1893)

(Figs 60-63)

1893 Chelyconus Montisclavus - SACco, p. 68, pl. 6, fig. 38, varieties: cappucinorum, pagodaeformis, inflatulospira, mamillatocrassa, angulatocrassa, humilispirata, magnomamillata, mamillospira: p. 68-69, pl. 6, figs 39-46.

1964 Conus montisclavus (Sacco) - HALL, p. 149, pl. 26, figs 1-3, 5-6 (cum syn.).

Material - 7 specimens - Coşteiu: M 60.9861.A-B., 60.9863.C.; Lăpugiu: M 60.8289.K., 60.8301.D., 68.599.C.; Nemeşeşti: M 60.8106.C.

Description - SL: 25-37. Spire low, outline convex, apex projected. Spiral whorls finely striate. Shoulder rounded. Body whorl conical, outline slightly sigmoid, smooth with fine ridges at the base. Subsutural flexure asymmetrically curved, shallow. Colour pattern of specimen M 68.599.C.: dense spiral rows of dashes.

Remarks - The species shows moderate variability of growth of the spire. Specimen M 68.599.C. matches V. montisclavus mamillospira (SAcco 1893, pl. 6, fig. 46), while specimen M 60.8301.D. is close to Chelyconus ponderosulcatus mamillatoides Sacco (refigured by Ferrero Mortara et al. 1984, pl. 19, fig. 8) that was considered as montisclavus by HALL (1964). This is the first record of the taxon from the Central Paratethys.

Distribution - Middle Miocene: Paratethys (Romania), Mediterranean (Italy). 


\section{Varioconus mucronatolaevis (Sacco, 1893)}

(Figs 64-65)

1893 Conus (Chelyconus) mucronatolaevis - SAcco, p. 66, pl. 6, fig. 26, varieties: fusoelegans, longovuloides, laevispira, taurobiconica, glandispira, globospira, permamillata, conicangulata: $\mathrm{p}$. 66-67, pl. 6, figs 27-34.

2014 Varioconus mucronatolaevis (Sacco) - Kovács \& Vicián, p. 85, figs 121-124 (cum syn.).

Materials - 4 specimens - Lăpugiu: M 60.8282.C-D., 68.599.C., 68.602.

Description - SL:28-30. Spire of moderate height, outline convex, apex projected. Spiral whorls smooth to finely striate. Shoulder rounded. Body whorl ventricosely conical, outline straight, smooth with fine ridges at the base. Subsutural flexure asymmetrically curved.

Remarks - The species is characterized by intraspecific variability with moderate variety of development of the spire. It differs from Plagioconus marii in lower and wider spire.

Distribution - Middle Miocene: Paratethys (Bulgaria, Hungary, Romania), Mediterranean (Italy).

\section{Varioconus noe (Brocchi, 1814)}

(Figs 66-68)

1814 Conus noe - Brocchi, p. 293, pl. 3, fig. 3.

1879 Conus (Chelyconus) Transsylvanicus nov. form. - Hoernes \& AUINGer, p. 41, pl. 1, fig. 14. 1997 Conus (Chelyconus) noe Brocchi - CHIRLI, p. 5, pl. 1, fig. 10 (cum syn.).

non 2001 Conus (Chelyconus) noe Brocchi - CHIRA \& VoiA, pl. 3, fig. 2. [= Varioconus clavatulus

(Orbigny, 1852)]

Material - 2 specimens - Lăpugiu: M 60.8358.E., 60.10156.

Description - SL: 51-60. Spire of moderate height, outline slightly convex. Spiral whorls high, slightly convex to concave, striate. Shoulder somewhat subangulate. Body whorl narrowly conical, smooth with fine ridges at the base. Subsutural flexure asymmetrically curved, of moderate depth. Colour pattern: dense spiral rows of dashes.

Remarks - V. clavatulus differs in smooth spiral whorls and in broader body whorl.

Distribution - Middle Miocene: Paratethys (Romania).

Varioconus olivaeformis (Hoernes et Auinger, 1879)

(Figs 69-70)

1879 Conus (Chelyconus) olivaeformis nov. form. - HoERnEs \& AUINGER, p. 52, pl. 1, fig. 23. 2014 Varioconus olivaeformis (Hoernes et Auinger) - Kovács \& Vicián, p. 85, figs 125-130. 

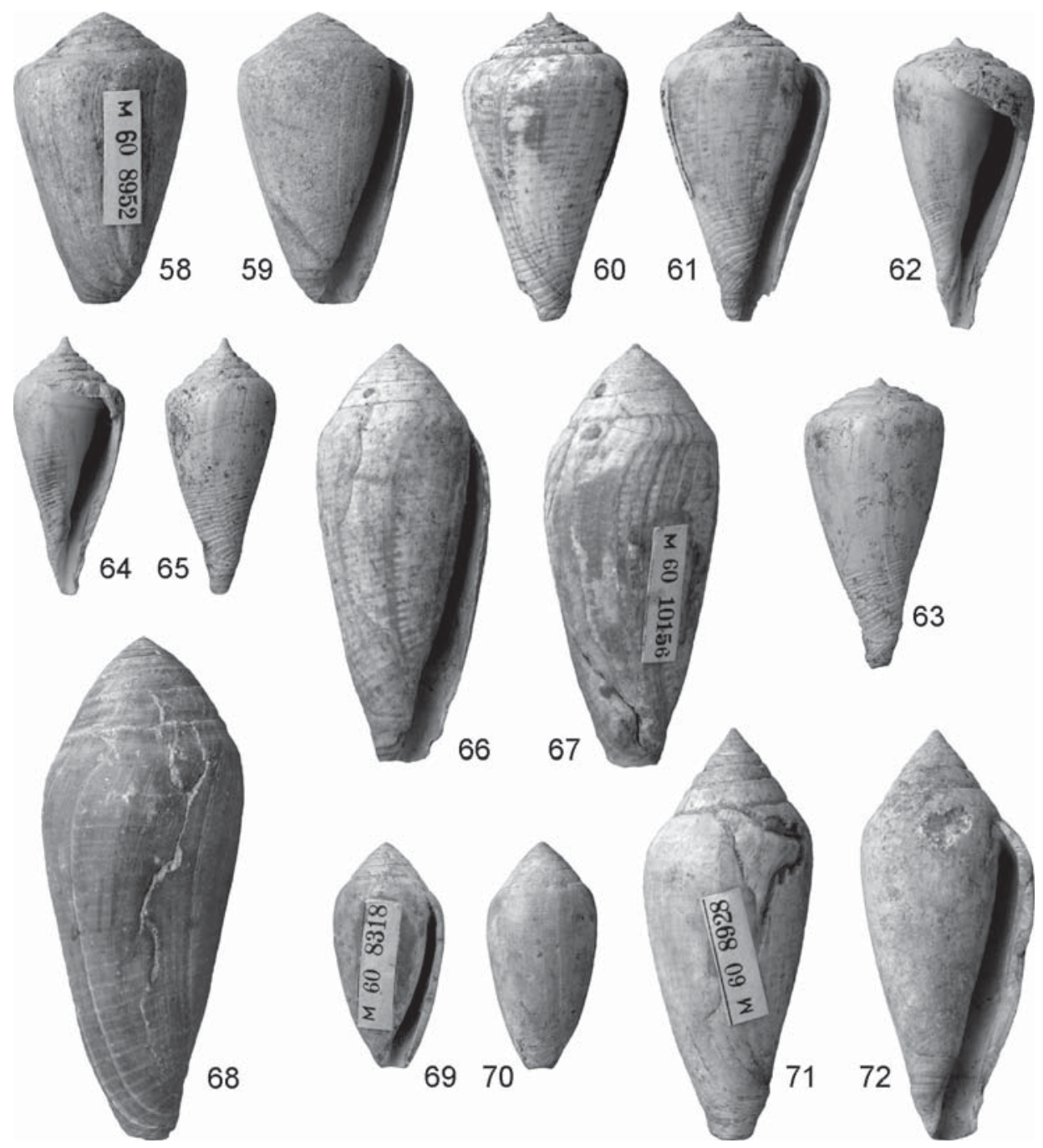

66
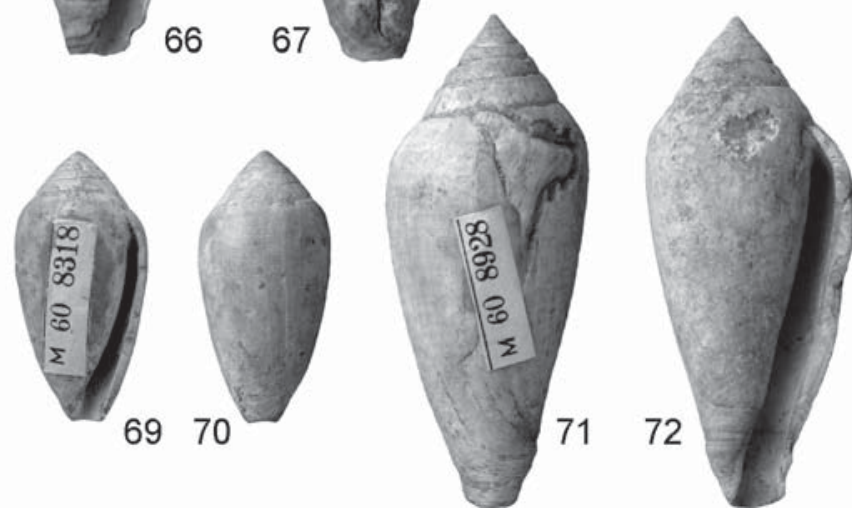

Figs 58-59. Varioconus conoponderosus (Sacco), M 60.8952., Coşteiu, SL: 34, abapertural and apertural views, (1×). - Figs 60-63. Varioconus montisclavus (Sacco): 60-61. M 68.599.C., Lăpugiu, SL: 37, abapertural and apertural views, (1×). 62-63. M 60.8301.D., Lăpugiu, SL: 35, apertural and abapertural views, (1×). - Figs 64-65. Varioconus mucronatolaevis (Sacco), M 60.8282.C., Lăpugiu, SL: 30, apertural and abapertural views, (1×). - Figs 66-68. Varioconus noe (Brocchi): 66-67. M 60.10156., Lăpugiu, SL: 50, apertural and abapertural views, (1×). 68. M 60.8358.E., Lăpugiu, SL: 60, abapertural view, (1x). - Figs 69-70. Varioconus olivaeformis (Hoernes et Auinger), M 60.8318.B., Lăpugiu, SL: 27, apertural and abapertural views, $(1 \times)$. - Figs 71-72. Varioconus pelagicus (Brocchi), M 60.8928., Lăpugiu, SL: 49, abapertural and apertural views, (1×) 
Material - 12 specimens - Coşteiu: M 60.9884.; Lăpugiu: M 60.8297.A-B., 60.8318.B-G., 60.10553.A-C.

Description - SL: 10-27. Spire moderately high, outline straight to slightly convex. Spiral whorls slightly convex, smooth. Shoulder rounded. Body whorl slightly elongate, narrowly ovate, outline convex, smooth with 4 spiral grooves at the base. Anterior notch slightly developed. Subsutural flexure asymmetrically curved.

Remarks - The species was abundant in the Letkés assemblage (KovÁcs \& VICIÁN 2014). It differs from V. ponderosus in size and in narrower shell.

Distribution - Middle Miocene: Paratethys (Bosnia, ?Czech Republic, Hungary, Romania).

Varioconus pelagicus (Brocchi, 1814)

(Figs 71-74)

1814 Conus pelagicus - Brocchi, p. 289, pl. 2, fig. 9.

1879 Conus (Chelyconus) Lapugyensis nov. form. - Hoernes \& Auinger, p. 42, pl. 1, fig. 9, pl. 5, fig. 8.

1879 Conus (Chelyconus) Mariae nov. form. - Hoernes \& Auinger, p. 49, pl. 6, fig. 7.

2013 Varioconus pelagicus (Brocchi) - LANDAU et al., p. 247, pl. 39, figs 6-7, pl. 41, fig. 14, pl. 42, fig. 8, pl. 82, figs 1-2 (cum syn.).

Material - 8 specimens - Coşteiu: M 68.17.; Lăpugiu: M 60.8281.A., 60.8296., 60.8314.A., 60.8928., 60.8939., 60.10182., 60.10201.

Description - SL: 22.5-64.5. Spire of moderate height, outline slightly convex to straight. Spiral whorls smooth to striate. Shoulder rounded. Body whorl conical, outline sigmoid, ornamented with fine spiral ridges on the lower half. Well-developed siphonal fasciole. Subsutural flexure nearly diagonal.

Remarks - The species shows variability of growth of the spire, and of length and width of the body whorl.

Distribution - Middle Miocene: Atlantic (France), Paratethys (Austria, Bulgaria, Croatia, Hungary, Romania, Ukraine), Mediterranean (Greece, Turkey).

\section{Varioconus ponderosus (Brocchi, 1814)}

(Figs 75-76)

1814 Conus ponderosus - BRocchI, p. 293, pl. 3, fig. 1.

2014 Varioconus ponderosus (Brocchi) - Kovács \& Vicián, p. 87, figs 138-142 (cum syn.).

Material - 39 specimens - Coşteiu: M 60.9863.B., 60.9871., 60.9874., 60.9876., 60.9880., 60.9898.A., 60.10104.; Lăpugiu: M 59.2521., 59.2004.A., 60.8282.B., 60.8288.A-E., 60.8292.A-F., 60.8294.A-B., 60.8304., 60.8306.A., 
60.8312.B-C., 60.8316.D., 60.8317.B., 60.8339.K-L., 60.8358.H., 60.9032.A-C., 60.9852.F-G., 64.241., 68.600.A.

Description - SL: 32-75. Spire of moderate height, outline straight. Whorls flat to convex, smooth. Shoulder subangulate to rounded. Body whorl conical, slightly convex, smooth with fine ridges at the anterior end. Aperture wide, somewhat flaring. Subsutural flexure asymmetrically curved.

Remarks - The species shows high level of intraspecific variability (HALL 1964, Davoli 1972).

Distribution - Middle Miocene: Atlantic (France), Paratethys (Austria, Bosnia, Czech Republic, Hungary, Moldavia, Poland, Romania, Serbia, Slovakia, Ukraine), Mediterranean (Greece, Turkey).

Varioconus praelongus (Hoernes et Auinger, 1879)

(Figs 77-78)

1879 Conus (Chelyconus) praelongus nov. form. - HoERnes \& AUINGER, p. 45, pl. 1, fig. 16.

1893 Chelyconus praelongus Hoernes et Auinger - SACco, p. 65.

1960 Conus (Chelyconus) praelongus Hoernes et Auinger - Kojumdgieva, p. 213, pl. 50, fig. 7.

? 1966 Conus (Chelyconus) praelongus fusiformis Halaváts - STRAUSZ, p. 461, text-fig. 208.

1967 Conus (Chelyconus) praelongus Hoernes et Auinger - DuşA, p. 57, pl. 2, fig. 9.

non 1985 Conus (Chelyconus) praelongus Hoernes et Auinger - ATANACKović, p. 178, pl. 39, figs

16-17 [= Varioconus olivaeformis (Hoernes et Auinger)]

Material: 3 specimens - Coşteiu: M 60.9863.A.; Lăpugiu: M 60.8314.B., 60.8358.F.

Description - SL: 22-30. Spire moderately high, outline slightly convex. Subapical whorls tuberculate, spiral whorls convex, striate. Shoulder rounded subangular. Body whorl conical, outline somewhat convex, smooth or ornamented with fine spiral ridges. Subsutural flexure asymmetrically curved, shallow.

Remarks - The type was described from Lăpugiu. V. pelagicus is a closely allied form, but differs in shell size and proportion, and in siphonal fasciole. The species shows variability of ornamentation. The shell of the type is wholly striate, but the striation of other specimens is weaker or absent. Based on new collecting works, the taxon can be recorded from Letkés. The specimen figured by Atanacković (1985) from Bosnia represents $V$. olivaeformis that differs from $V$. praelongus in lower spire and in ovate body whorl.

Distribution - Middle Miocene: Paratethys (Austria, Bulgaria, ?Poland, Romania). 
Varioconus pseudoponderosus (Glibert, 1952)

(Figs 79-80)

1952 Conus (Chelyconus) pseudoponderosus Dollfus et Dautzenberg mss., nov. sp. - GLIBERT, p. 376, pl. 13, fig. 4.

1966 Conus (Chelyconus) pseudoponderosus Dollfus et Dautzenberg (in Glibert) - STRAusz, p. 463 , pl. 68, figs 6-7.

1973 Conus (Chelyconus) pseudoponderosus Glibert - Boнn-Havas, p. 1122, pl. 7, fig. 4, pl. 9, fig. 12.

2010 Conus pseudoponderosus Glibert - VAessen, p. 12, figs 10/C, 15-17, 18/B.

Material - 2 specimens - Coşteiu: M 60.9878.B.; Lăpugiu: M 60.8958.

Description - SL: 47-48.5. Spire moderately high, outline straight. Spiral whorls convex, smooth, suture deep. Shoulder rounded. Body whorl conical, outline straight, smooth with fine spiral ridges at the base. Subsutural flexure asymmetrically curved, of moderate depth.

Remarks - This rare species was regarded as a synonym of $V$. ponderosus by Hall (1964) and by Kovács \& Vicián (2014). However, the morphological features allow acceptance of the validity, so here we follow the recent classification of VAESSEN (2010). V. pseudoponderosus differs from both $V$. clavatulus and $V$.ponderosus in lower spire with straight outline and in broader shell. The species is probable a transitional form between $V$. ponderosus and $V$. conoponderosus.

Distribution - Middle Miocene: Atlantic (France), Paratethys (Hungary, Romania).

\section{Varioconus steinabrunnensis (Sacco, 1893)}

(Figs 81-82)

1893 Conus (Chelyconus) steinabrunnensis - SACco, p. 75.

2014 Chelyconus steinabrunnensis Sacco - Kovács \& Vicián, p. 61, figs 22-27 (cum syn.).

Material - 21 specimens - Coşteiu: M 60.9854.A-B.; Lăpugiu: M 60.8282.B., 60.8284., 60.8289.J., 60.8290.N., 60.8294.D., 60.8337.D-F., 60.8343.B-D., 60.8969.E-F., 60.10117.B., 60.10311.B., 64.392.B., 68.595.L-M., 68.599.B.

Description - SL: 15.5-47. Spire low, outline concave. Whorls smooth, ramps flat. Shoulder angulate. Body whorl conical, outline convex, smooth with fine grooves at the base. Subsutural flexure asymmetrically curved.

Remarks - As genus Chelyconus was not accepted for fossil conoids by TUCKer \& TENORIO (2009) and LANDAU et al. (2013), we place steinabrunnensis within genus Varioconus herein. The species differs from Kalloconus fuscocingulatus in angulate shoulder, from $V$. vindobonensis in lower spire, and from $V$. karamanensis (Erünal-Erentöz) in striate spire. 


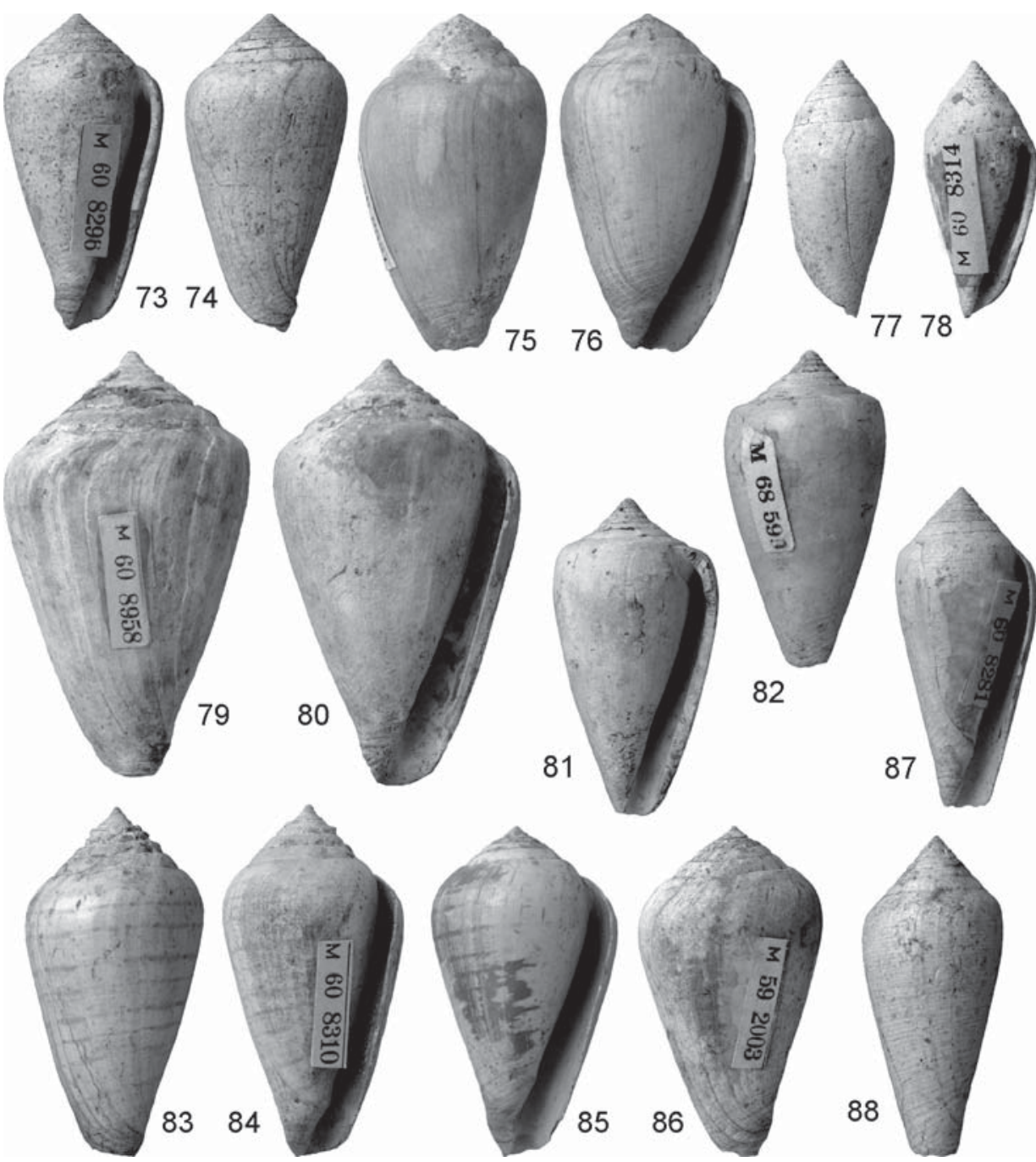

Figs 73-74. Varioconus pelagicus (Brocchi), M 60.8296., Lăpugiu, SL: 37, apertural and abapertural views, $(1 \times)$. - Figs 75-76. Varioconus ponderosus (Brocchi), M 59.2521., Lăpugiu, SL: 39, abapertural and apertural views, $(1 \times)$. - Figs 77-78. Varioconus praelongus (Hoernes et Auinger), $M$ 60.8314.B., Lăpugiu, SL: 30, abapertural and apertural views, (1×). - Figs 79-80. Varioconus pseudoponderosus (Glibert), M 60.8958., Lăpugiu, SL: 49, abapertural and apertural views, (1×). - Figs 81-82. Varioconus steinabrunnensis (Sacco), M 68.599.B., Lăpugiu, SL: 37, apertural and abapertural views, $(1 \times)$. - Figs 83-86. Varioconus subraristriatus (da Costa): 83-84. M 60.8310., Lăpugiu, SL: 41, abapertural and apertural views, (1×). 85-86. M 59.2003., Lăpugiu, SL: 39, apertural and abapertural views, (1×). - Figs 87-88. Varioconus suessi (Hoernes et Auinger), M 60.8281.B., Lăpugiu, SL: 38 , apertural and abapertural views, $(1 \times)$ 
Distribution - Middle Miocene: Paratethys (Austria, Bosnia, Bulgaria, Hungary, Poland, Romania).

Varioconus subraristriatus (da Costa, 1866)

(Figs 83-86)

1866 Conus subraristriatus Costa - DA CosTA, p. 15, pl. 4, figs 2, 7 only.

1966 Conus (Cleobula) subraristriatus - STRAUSz, p. 465, pl. 71, figs 3-5.

1973 Conus (Dendroconus) subraristriatus Costa - BoHn-Havas, p. 1070, pl. 7, figs 1-2, pl. 9, fig. 11.

2013 Varioconus subraristriatus (Pereira da Costa) - LANDAU et al., p. 250, pl. 40, figs 7-8, pl. 41, fig. 17, pl. 42, fig. 11.

2014 Varioconus enzesfeldensis (Hoernes et Auinger) - KovÁcs \& VICIÁN, p. 84, figs 3, 116-120 (cum syn.).

Material - 15 specimens - Coşteiu: M 60.9872., 60.10179., 68.19.; Lăpugiu: M 59.1840., 59.2003., 60.7676., 60.8310., 60.8335.L., 60.8336.H., 60.8337.M-N., 60.8345.A-D.

Description - SL: 28-44. Spire of moderate height, outline slightly convex, apex projected. Spiral whorls slightly convex, smooth. Shoulder rounded. Body whorl broadly ovate, smooth with fine grooves at the base. Subsutural flexure nearly diagonal. Colour pattern: widely spaced spiral rows of brownish dashes.

Remarks - V. enzesfeldensis (Hoernes et Auinger) was regarded as a synonym of $V$. subraristriatus by LANDAU et al. (2013). The species shows moderate variability in the height of the spire.

Distribution - Middle Miocene: Paratethys (Austria, Bosnia, Hungary, Romania), Mediterranean (Greece, Turkey).

Varioconus suessi (Hoernes et Auinger, 1879)

(Figs 87-88)

1879 Conus (Chelyconus) Suessi nov. form. - Hoernes \& AUINGER, p. 43, pl. 1, figs 1, 15, pl. 6, figs

1-2 only.

1960 Conus (Chelyconus) suessi Hoernes et Auinger - Kojumdgieva, p. 212, pl. 49, fig. 8.

1966 Conus (Chelyconus) suessi Hoernes et Auinger - Strausz, p. 459, pl. 69, fig. 6.

1976 Conus (Chelyconus) suessi Hoernes et Auinger - PAPP, p. 136, pl. 35, figs 16-17, pl. 36, figs 6-7.

Material - 12 specimens - Coşteiu: M 60.10105.; Lăpugiu: M 60.8281.B-C., 60.8292.G-H., 60.8307., 60.8358.A-D., 60.8360.E., 60.10148.

Description - SL: 28-85. Spire of moderate height, outline straight. Spiral whorls flat, smooth. Shoulder rounded. Body whorl conical, outline straight to slightly convex, ornamented with fine spiral ridges. Subsutural flexure asymmetrically curved, shallow. 
Remarks - The taxon was considered as a junior synonym of $V$. pelagicus by KovÁcs \& Vicián (2014). After the analysis of the Făget Basin materials, however, we recognize the validity of the species. V. pelagicus differs in sigmoid body whorl with well-developed siphonal fasciole, and in nearly diagonal subsutural flexure.

Distribution - Middle Miocene: Paratethys (Bulgaria, Hungary, Romania).

Varioconus taurinensis (Bellardi et Michelotti, 1840)

(Figs 89-90)

1840 Conus Striatulus Brocchi var. Taurinensis - Bellardi \& Michelotti, p. 62, pl. 7, figs 12-13.

2013 Varioconus taurinensis (Bellardi et Michelotti) - LANDAU et al., p. 251, pl. 41, figs 4-6, 19, pl. 42, fig. 13, pl. 82, figs 6-7.

2014 Varioconus taurinensis (Bellardi et Michelotti) - Kovács \& Vicián, p. 88, figs 143-148.

Material - 1 specimen - Lăpugiu: M 60.8339.O.

Description - SL: 15.5. Spire irregular, variable, mamillate to high. The ultimate spiral whorl is higher than the preceding whorls, convex to step-like. Shoulder sloping. Body whorl conical, outline convex, smooth with fine ridges at the base. Subsutural flexure nearly diagonal.

Remarks - The species was relatively abundant in the Letkés assemblage (KovÁcs \& Vicín 2014).

Distribution - Middle Miocene: Paratethys (Hungary, Romania), Mediterranean (Albania, Turkey).

Varioconus taurorectus (Sacco, 1893)

(Figs 91-92)

1893 Chelyconus taurorectus - SACCo, p. 67, pl. 6, fig. 35, variety: proappenninica: p. 68, pl. 6, fig. 37. 1964 Conus taurorectus (Sacco) - Hall, p. 161, pl. 20, figs 8, 13.

Material - 1 specimen - Lăpugiu: M 60.8355.

Description - SL: 55. Spire of moderate height, outline straight. Spiral whorls slightly convex, smooth, suture deep. Shoulder broad, rounded. Body whorl broadly conical, with prominent growth lines. Aperture somewhat flaring at the base. Subsutural flexure asymmetrically curved, deep.

Remarks - The specimen slightly differs from the type (refigured by FERRERO MORTARA et al. 1984, pl. 18, fig. 4) in narrower base. The species is probably a morphotype of $V$. conoponderosus.

Distribution - Middle Miocene: Paratethys (Romania). 
Varioconus vindobonensis (Hörnes, 1856)

(Figs 93-94)

1879 Conus (Chelyconus) vindobonensis Partsch - Hoernes \& Auinger, p. 48.

non 2001 Conus (Chelyconus) vindobonensis Partsch in Hörnes - CHira \& VoiA, pl. 2, fig. 5. [= ? Varioconus ponderosus (Brocchi)]

2014 Chelyconus vindobonensis (Partsch in Hörnes) - KovÁcs \& Vicián, p. 62, figs 28-29 (cum syn.).

Material - 21 specimens - Coşteiu: M 68.16.; Lăpugiu: M 60.8277.B-D., 60.8297.F., 60.8301.E., 60.8306.B., 60.8313.A-E., 60.8317.D-F., 60.8345.E-F., 60.8346.B., 60.8353.K-M., 60.8941.B.

Description - SL: 13-38. Spire of moderate height, outline concave. Protoconch multispiral, projected. Spiral whorls convex, smooth. Shoulder angulate. Body whorl conical, outline convex, smooth with fine grooves at the base. Subsutural flexure asymmetrically curved.

Remarks - V. steinabrunnensis is a similar form but differs in more elongate body whorl and in lower spire with flat whorls.

Distribution - Middle Miocene: Paratethys (Austria, Czech Republic, Hungary, Moldavia, Poland, Romania, Serbia), Mediterranean (Turkey).

Genus Conilithes Swainson, 1840

Conilithes antidiluvianus (Bruguière, 1792)

(Figs 95-96)

1792 Conus antidiluvianus - BRUGUì̀re, p. 637, pl. 347, fig. 6.

1879 Conus (Leptoconus) Berwerthi nov. form. - Hoernes \& AUINGER, p. 35, pl. 5, figs 11-12.

2014a Conilithes antidiluvianus (Bruguière) - JANSSEN et al., p. 73-90.

Material - 7 specimens - Coşteiu: M 60.8924.A-C., 60.9873.A., 60.9876.; Lăpugiu: M 60.8285., 60.8922.

Description - SL: 6-46. Spire high, outline straight, step-like. Sutural ramps concave, smooth. Shoulder carinate, finely tuberculate. Body whorl conical, outline straight, smooth with fine spiral grooves at the anterior end. Subsutural flexure asymmetrically curved.

Remarks - A comprehensive revision of the taxon was recently provided by JANSSEN et al. $(2014 a, b)$.

Distribution - Middle Miocene: Atlantic (Portugal), North Sea (Belgium, Denmark, Germany, the Netherlands), Paratethys (Austria, Bosnia, Bulgaria, Czech Republic, Hungary, Poland, Romania), Mediterranean (Albania, Greece, Italy, Morocco, Turkey). 


\section{Conilithes brocchii (Bronn, 1828)}

(Figs 97-98)

1828 Conus Brocchii - Bronn, p. 740.

2014 Conilithes brocchii (Bronn) - Kovács \& ViCián, p. 89, figs 150-151 (cum syn.).

Material - 9 specimens - Coşteiu: M 60.9870., 60.9882.; Lăpugiu: M 60.8276.A-B., 60.8317.A., 60.8319.A-B., 60.8933., 60.10595.B.

Description - SL: 14-16. Spire of low to moderate height, conical, outline concave. Whorls finely striate with raised edge. Weakly beaded apical whorls. Shoulder angulate. Body whorl elongate conical, outline straight. Subsutural flexure asymmetrically curved, of moderate depth.

Remarks - C. striatulus is a similar form but differs in flat and smooth spiral whorls.

Distribution - Middle Miocene: Paratethys (Austria, Hungary, Romania), Mediterranean (Greece, Italy).

\section{Conilithes canaliculatus (Brocchi, 1814)}

(Figs 99-104)

1814 Conus canaliculatus - Brocchi, p. 636, pl. 15, fig. 28.

1879 Conus (Leptoconus) Brezinae nov. form. - HoERnEs \& AUINGER, p. 36.

? 1887 Conus (Chelyconus) sceptophorus n. sp. - BOETtger, p. 7, pl. 2, figs 6-8.

2013 Conilithes dujardini (Deshayes) - LANDAU et al., p. 252, pl. 41, figs 1-3, 18, pl. 42, fig. 12, pl.

82 , fig. 5.

2014 Conilithes canaliculatus (Brocchi) - Kovács \& VicıÁN, p. 91, figs 152-156 (cum syn.).

Material - 56 specimens - Coşteiu: M 60.8966., 60.9866., 60.9887.A-D., 60.9890.A-C.; Lăpugiu: M 60.6335., 60.8276.D-I., 60.8297.C-E., 60.8305. (17 spp.), 60.8319.F-I., 60.8323.A-H., 60.8335.M., 60.8941.A., 60.8967., 60.10075.AB., 68.596.A., 68.606.A-B.

Description - SL: 6-37. Shell biconical, spire elevated, outline straight. Spiral whorls high, angular, dropped, smooth or striate, weakly tuberculate near the apex. Sutural ramps steep, slightly concave. Shoulder angulate, sloping. Body whorl smooth with incised grooves at the anterior end. Aperture straight, narrow. Subsutural flexure symmetrically curved.

Remarks - Taxonomical problems of the species was recently treated by Kovács \& Vicián (2014). C. dujardini differs markedly in morphology (see below).

Distribution - Middle Miocene: Paratethys (Austria, Bosnia, Bulgaria, Czech Republic, Hungary, Poland, Romania, Serbia, Slovakia, Slovenia, Ukraine), Mediterranean (Greece, Italy, Turkey). 
Conilithes dujardini (Deshayes, 1845)

(Figs 105-108)

1845 Conus Dujardini - DeshaYes, p. 158.

2014 Conilithes dujardini (Deshayes) - Kovács \& ViCıÁN, p. 92, figs 157-159 (cum syn.).

Material - 220 specimens - Coşteiu: M 60.7540., 60.8934., 60.8940.AG., 60.9864., 60.9866., 60.9868. (22 spp.), 60.9873.C-I., 60.9875., 60.9885.AE., 60.9887. (22 spp.), 60.9889., 60.9898.A-C., 60.10070.A-B., 60.10276.AD.; Lăpugiu: M 60.7739.A-F., 60.8276. (39 spp.), 60.8308.A-C., 60.8319.J-S., 60.8323.I-J., 60.8340. (49 spp.), 60.8919.E-F., 60.8921.A-G., 60.8925., 60.8936., 68.595.A-K., 68.600.B., 68.596.B-C., 68.607.A-B.; Nemeşeşti: M 60.8108.A-E., 60.8118.

Description - SL: 5-38. Spire high, outline straight to concave. Apical whorls tuberculate. Spiral whorls smooth to finely striate. Shoulder angulate in early whorls, carinate in the last whorl. Body whorl conical, outline concave, either smooth with spiral grooves from the base up to the mid-height or fully ornamented with fine grooves. Subsutural flexure asymmetrically curved, deep. Colour pattern: spiral rows of small dots.

Remarks - Taxonomical problems of the species was recently discussed by Kovács \& Vicián (2014). The identity of $C$. dujardini and C. exaltatus (Eichwald) requires further research. In the Făget Basin assemblages the proportion of fully ornamented specimens is lower than in the Letkés material. The ornamentation shows fine gradation from smooth to fully ornamented body whorl in the material described here. $C$. canaliculatus differs in shell proportion, growth of the spire, in less raised and inclined spiral whorls, non carinate shoulder, and in colour pattern (see CAZE et al. 2010, fig. 5/N, LANDAU et al. 2013, pl. 82, fig. 5, and Paganelli 2014, pl. 5, fig. 3).

Distribution - Middle Miocene: Atlantic (France, Portugal), North Sea (Belgium, Denmark, Germany, the Netherlands), Paratethys (Austria, Bosnia, Bulgaria, Croatia, Czech Republic, Hungary, Moldavia, Poland, Romania, Serbia, Slovakia, Slovenia, Ukraine), Mediterranean (Greece, Italy, Libya, Turkey).

Conilithes granularis (Borson, 1820)

(Figs 109-110)

1820 Conus Granularis - Borson, p. 196, pl. 1, fig. 3.

1879 Conus (Stephanoconus) Stachei nov. form. - Hoernes \& AUINGER, p. 16, pl. 6, figs 14-16.

1902 Conus (Stephanoconus) wagneri n. sp. - BOettger, p. 7.

2014 Conilithes granularis (Borson) - Kovács \& Vicián, p. 93, fig. 160 (cum syn.). 


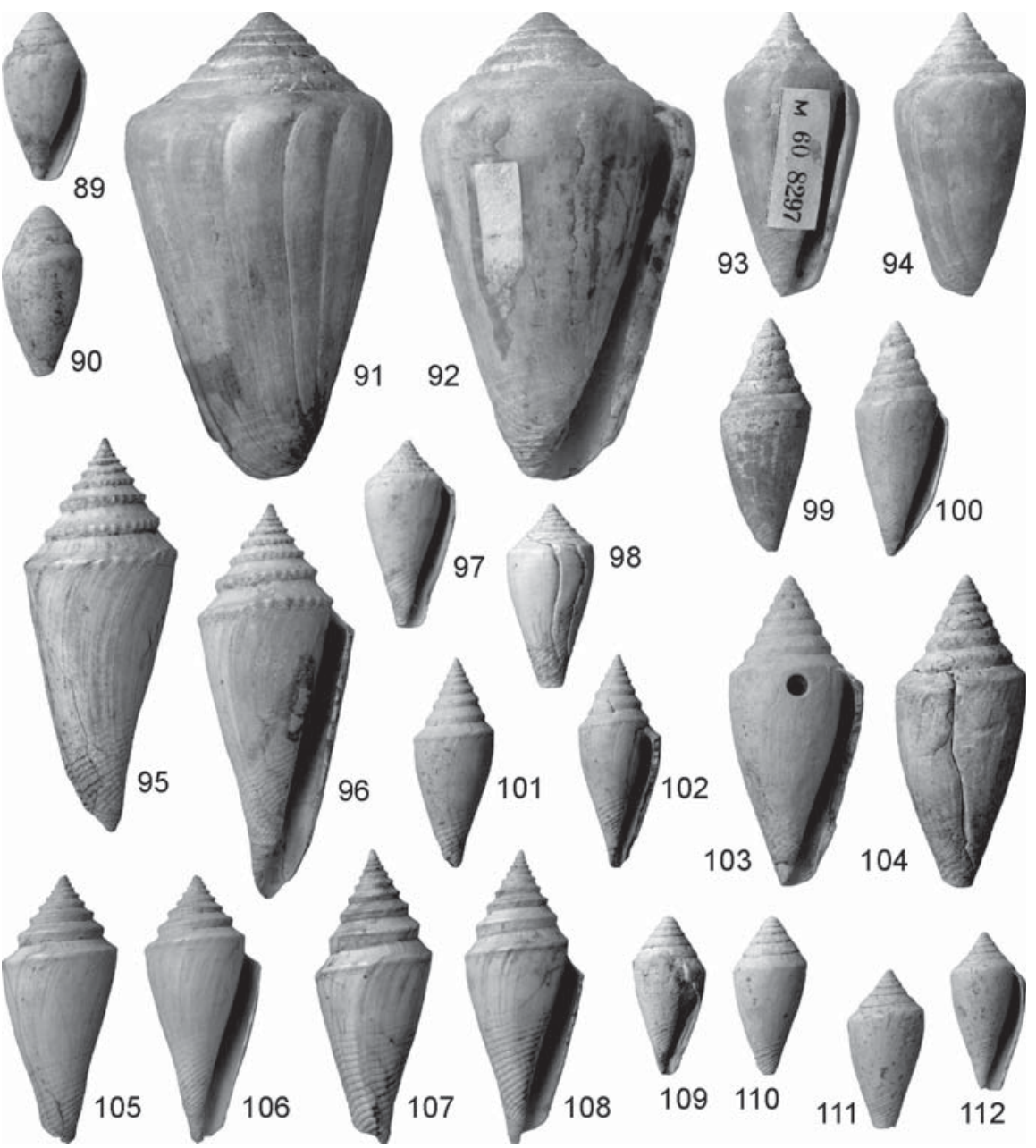

Figs 89-90. Varioconus taurinensis (Bellardi et Michelotti), M 60.8339.O., Lăpugiu, SL: 15.5, apertural and abapertural views, (1.25×). - Figs 91-92. Varioconus taurorectus (Sacco), M 60.8355., Lăpugiu, SL: 55, abapertural and apertural views, $(1 \times)$. - Figs 93-94. Varioconus vindobonensis (Hörnes), M 60.8297.F., Lăpugiu, SL: 33, apertural and abapertural views, (1×). - Figs 95-96. Conilithes antidiluvianus (Bruguière), M 60.9876., Coşteiu, SL: 46, abapertural and apertural views, (1×). - Figs 97-98. Conilithes brocchii (Bronn), M 60.8276.A., Lăpugiu, SL: 17, abapertural and apertural views, (1.25×). - Figs 99-104. Conilithes canaliculatus (Brocchi): 99-100. M 60.8323A., Lăpugiu, SL: 26.5, abapertural and apertural views, (1×). 101-102. M 60.8323.B., Lăpugiu, SL: 24, abapertural and apertural views, (1×). 103-104. M 60.9890.A., Coşteiu, SL: 36, apertural and abapertural views, (1×). - Figs 105-108. Conilithes dujardini (Deshayes): 105-106. M 60.9868.B., Coşteiu, SL: 31, abapertural and apertural views, (1×). 107-108. M 60.9868.A., Coşteiu, SL: 34, abapertural and apertural views, $(1 \times)$. - Figs 109-110. Conilithes granularis (Borson), M 60.9863.D., Coşteiu, SL: 12, abapertural and apertural views, (1.5 $\times$ ). - Figs 111-112. Conilithes striatulus (Brocchi), M 608305.A., Lăpugiu, SL: 12.5, abapertural and apertural views, $(1.5 \times)$ 
Material - 221 specimens - Coşteiu: M 60.8926. (39 spp.), 60.9853.A-B., 60.9855.A-D., 60.9860.A-B., 60.9862.C., 60.9863.D-E., 60.9866. (153 spp.), 60.9867.A-B., 60.9869., 60.9870., 60.9875., 60.9881.A-E., 60.9886., 60.10276.E-I.; Lăpugiu: M 60.10553.D., 68.595.N.

Description - SL: 4-12.5. Shell biconical, spire high, outline straight, whorls ornamented with two fine grooves in the middle. Shoulder angular. Body whorl conical, outline straight to sigmoid, smooth with spiral ridges at the base or entirely covered with regularly spaced, fine spiral ridges. Subsutural flexure symmetrically curved, of moderate depth.

Remarks - The species is characterised by two phenotypically different forms with granulate or striate body whorl. As size and morphology of "Stephanoconus" wagneri Boettger agree well with that of $C$. granularis, it is considered here as a junior synonym.

Distribution - Middle Miocene: Paratethys (Austria, Bosnia, Bulgaria, Czech Republic, Hungary, Poland, Romania, Slovakia, Ukraine), Mediterranean (Italy).

Conilithes striatulus (Brocchi, 1814)

(Figs 111-112)

1814 Conus striatulus - Brocchi, p. 294, pl. 3, fig. 4.

2003 Conus striatulus Brocchi - Davoli, p. 453, pl. 1, figs 2, 5, pl. 2, fig. 14.

Material - 5 specimens - Lăpugiu: M 60.8305.A-B., 60.8319.C-E.

Description - SL: 12-15. Spire of moderate height, conical, outline straight. Whorls smooth with slightly raised edge. Shoulder angulate. Body whorl conical, outline straight, smooth with spiral ridges at the base. Subsutural flexure asymmetrically curved.

Remarks - C. brocchii differs in striate spiral whorls. Based on new collecting works the occurrence of the species can be recorded from Letkés.

Distribution - Middle Miocene: Paratethys (Hungary, Romania).

\section{CONCLUSION}

Based on taxonomical revision, 41 conoid species are described here from three Early Badenian localities of the Făget Basin in Romania. Some of them (e.g. L. eschewegi, V. montisclavus or $V$. taurorectus) are new records from the region. From Lăpugiu, 37 species are represented by 579 specimens with dominance of C. dujardini (136 spp.), L. bitorosus (62), K. berghausi (52), and C. canaliculatus (47). From Coşteiu, 30 species are represented by 372 specimens with dominance of C. granularis (219) and C. dujardini (78). This difference can be explained by diverse origins of the museum collection (collecting works, donations, acquisi- 
tion), as sediments of the same age are very similar at both localities. The small material from Nemeşeşti contains only 14 specimens that represent 9 species.

A few taxa that were recorded from Lăpugiu in earlier papers are absent from this museum collection, consequently the synthesis of the Conidae fauna of the region requires more research. To understand the actual diversity of this material, the comprehensive taxonomic revision of the Central Paratethyan Conidae is also inevitable. Some species assigned by Hoernes \& AUINGer (1879) or SACCo (1893) were revised by HALL (1964) and by LANDAU et al. (2013), a few taxa are revised here, but others obviously need reinterpretation. Until further works we do not take account of records of e.g. "Conus" neumayri Hoernes et Auinger, "Conus" karreri Hoernes et Auinger, "Conus" schroeckingeri Hoernes et Auinger recorded by $\mathrm{KocH}$ (1900), and "Stephanoconus" subnocturnus (Orbigny) recorded by CHIRA \& Voia (2001) from the region.

On the other hand, the fauna list of Lăpugiu is completed here with four other taxa. Conus subcoronatus was described from this locality by BoETTGER (1887). The species was cited in subsequent papers (e.g. КосH 1900), but it was never found again. Boettger's collection is stored in Frankfurt, and we could study the photos of the holotype by courtesy of Dr. Ronald Janssen (Senckenberg Forschungsinstitut). The morphology of subcoronatus is close to Pseudonoduloconus gastaldii (Michelotti) in nodulose spiral whorls and shoulder, however, it differs by convex, not canaliculate spiral whorls. Based on these features, the validity of the species is recognized in this paper, and classified within genus Pseudonoduloconus Tucker et Tenorio.

Furthermore, taking notice of questionable synonymies, occurrences of three other taxa at Lăpugiu are accepted here from the records of CHIRA \& VoIA (2001): Kalloconus betulinoides (Lamarck), Monteiroconus antiquus (Lamarck), and Varioconus raristriatus (Bellardi et Michelotti). These species and P. subcoronatus are indicated by * in Table 1.

From the Central Paratethys, the rich material presented here can be compared with that of the Börzsöny Mts (N Hungary). The Conidae fauna from Szob and Letkés also shows high diversity with 45 species (CSEPREGHY-MEZNERICS 1956, STrausz 1966, Kovács \& ViCián 2014). The assemblages of the two regions differ in the proportions of taxa. The dominant taxa at the Letkés section were L. bitorosus, C. canaliculatus, V. miovoeslauensis, and $K$. berghausi, while in the Făget Basin C. granularis, C. dujardini, L. bitorosus, and K. berghausi prevail in the fauna. Altogether 51 Conidae species occur in the two regions, the proportion of shared taxa is $78.4 \%$. Both assemblages are of mixed composition, they are related to the Conidae faunas known from the Vienna Basin and Northern Italy. According to Harzhauser \& Piller (2007) a clear Mediterranean character became dominant in the Central Paratethys in the early Middle Miocene. Our 
Table 1. Conidae species from the Făget Basin (SW Romania) and from the Börzsöny Mts (N Hungary). ${ }^{*}$ indicates taxa that are absent from the collection of the Hungarian Natural History Museum, Budapest, but known from the papers of Boettger (1887) and Chira \& VoiA (2001). ** indicates new, unpublished Conidae records from Letkés (Börzsöny Mts)

\begin{tabular}{|c|c|c|}
\hline species & Făget Basin & Börzsöny Mts \\
\hline aldrovandi, Monteiroconus (Brocchi) & - & 0 \\
\hline anguliferus, Monteiroconus cf. (Peyrot) & - & $\circ$ \\
\hline antidiluvianus, Conilithes (Bruguière) & $\circ$ & ० \\
\hline antiquus, Monteiroconus (Lamarck) & * & ○ \\
\hline berghausi, Kalloconus (Michelotti) & ○ & ○ \\
\hline betulinoides, Kalloconus (Lamarck) & * & ○ \\
\hline bitorosus, Lautoconus (Fontannes) & o & o \\
\hline brocchii, Conilithes (Bronn) & $\circ$ & $\circ$ \\
\hline canaliculatus, Conilithes (Brocchi) & $\circ$ & ० \\
\hline clavatulus, Varioconus (d'Orbigny) & $\circ$ & $\circ$ \\
\hline conoponderosus, Varioconus (Sacco) & $\circ$ & ○ \\
\hline daciae, Kalloconus (Hoernes et Auinger) & $\circ$ & $\circ$ \\
\hline dertogibbus, Varioconus (Sacco) & - & $\circ$ \\
\hline dujardini, Conilithes (Deshayes) & $\circ$ & ○ \\
\hline elongatus, Plagioconus (Borson) & o & o \\
\hline eschewegi, Lautoconus (da Costa) & ○ & o \\
\hline extensus, Plagioconus (Partsch in Hörnes) & ० & $\circ$ \\
\hline fuscocingulatus, Kalloconus (Bronn in Hörnes) & $\circ$ & $\circ$ \\
\hline granularis, Conilithes (Borson) & $\circ$ & ० \\
\hline hirmetzli, Leptoconus Kovács et Vicián & - & $\circ$ \\
\hline hungaricus, Kalloconus (Hoernes et Auinger) & $\circ$ & ○ \\
\hline marii, Plagioconus (Sacco) & $\circ$ & ० \\
\hline mercati, Monteiroconus (Brocchi) & o & o \\
\hline miovoeslauensis, Lautoconus (Sacco) & o & o \\
\hline mojsvari, Monteiroconus (Hoernes et Auinger) & $\circ$ & $\circ$ \\
\hline montisclavus, Varioconus (Sacco) & $\circ$ & - \\
\hline mucronatolaevis, Varioconus (Sacco) & $\circ$ & $\circ$ \\
\hline noe, Varioconus (Brocchi) & $\circ$ & - \\
\hline oboesus, Varioconus (Michelotti) & - & ** \\
\hline olivaeformis, Varioconus (Hoernes et Auinger) & $\circ$ & ० \\
\hline parvicaudatus, Monteiroconus (Sacco) & - & ० \\
\hline pelagicus, Varioconus (Brocchi) & $\circ$ & $\circ$ \\
\hline ponderosus, Varioconus (Brocchi) & $\circ$ & $\circ$ \\
\hline praelongus, Varioconus (Hoernes et Auinger) & $\circ$ & ** \\
\hline pseudoponderosus, Varioconus (Glibert) & $\circ$ & $\circ$ \\
\hline puschi, Plagioconus (Michelotti) & $\circ$ & $\circ$ \\
\hline pyrula, Lautoconus (Brocchi) & $\circ$ & o \\
\hline raristriatus, Varioconus (Bellardi et Michelotti) & * & ० \\
\hline rotundus, Lautoconus (Hoernes et Auinger) & $\circ$ & ० \\
\hline steinabrunnensis, Varioconus (Sacco) & $\circ$ & ○ \\
\hline steindachneri, Kalloconus (Hoernes et Auinger) & $\circ$ & ० \\
\hline striatulus, Conilithes (Brocchi) & $\circ$ & ** \\
\hline subbigranosus, Pseudonoduloconus cf. (Sacco) & $\circ$ & - \\
\hline subraristriatus, Varioconus (da Costa) & $\circ$ & $\circ$ \\
\hline subcoronatus, Pseudonoduloconus (Böttger) & * & - \\
\hline suessi, Varioconus (Hoernes et Auinger) & $\circ$ & o \\
\hline taurinensis, Varioconus (Bellardi et Michelotti) & $\circ$ & $\circ$ \\
\hline taurorectus, Varioconus (Sacco) & $\circ$ & - \\
\hline tietzei, Monteiroconus (Hoernes et Auinger) & ○ & o \\
\hline ventricosus, Lautoconus (Gmelin) & $\circ$ & ० \\
\hline vindobonensis, Varioconus (Partsch in Hörnes) & 으 & 으 \\
\hline
\end{tabular}


achievements also prove a close relationship between the Central Paratethyan and the Mediterranean domains during the Langhian.

Acknowledgements - The Conidae specimens were kindly offered for study to us by Alfréd Dulai (Budapest). A. Dulai's and Ronald Janssen's (Frankfurt) constructive reviews helped to improve the manuscript. R. Janssen kindly sent us photos of Boettger's type specimens. Lóránd Silye (Cluj) and Manuel Tenorio (Cádiz) supported our work with reference books. Olga Piros (Budapest) helped to prepare the map of the localities. We are also grateful to the staff of the Geological Library of the Geological and Geophysical Institute of Hungary.

\section{REFERENCES}

Atanacković M. A. 1985: Mekušci morskog miocena Bosne. - Geoinženjering, Sarajevo, 308 pp.

BA£UK W. 1997: Middle Miocene (Badenian) gastropods from Korytnica, Poland; Part III. - Acta Geologica Polonica 47(1-2): 3-75.

BAŁUK W. 2006: Middle Miocene (Badenian) gastropods from Korytnica, Poland; Part V. Addenda et corrigenda ad Prosobranchia. - Acta Geologica Polonica 56(2): 177-220.

Bellardi L. \& Michelotti G. 1840: Saggio orittografico sulla classe dei gasteropodi fossili dei terreni terziarii del Piemonte. - Tipografia Reale, Torino, 80 pp.

Boettger O. 1887: Drei neue Conus aus dem Miocaen von Lapugy und von Bordeaux. - Jahrbücher der Deutschen Malakozoologischen Gesellschaft 14: 4-8.

Boettger O. 1897: Zur Kenntnis der Fauna der mittelmiozänen Schichten von Kostej im Banat. - Verhandlungen und Mitteilungen des siebenbürgischen Vereines für Naturwissenschaften zu Hermannstadt 46(1896): 49-66.

Boettger O. 1902-1907: Zur Kenntnis der Fauna der mittelmiozänen Schichten von Kostej im Krassó-Szörényer Komitat. - Verhandlungen und Mitteilungen des siebenbürgischen Vereines für Naturwissenschaften zu Hermannstadt 51(1902): 1-200, 54(1906): 1-99, 55(1907): 101-244.

Bohn-Havas M. 1973: A Keleti-Mecsek torton Mollusca faunája. (Tortonische Molluskenfauna des Östlichen Mecsek-Gebirges.) - Jahrbuch der Ungarischen Geologischen Anstalt 53(4): 951-1079, (1081-1161).

Borson S. 1820: Saggio di Orittografia Piemontese. - Memorie della Reale Accademia delle Scienze di Torino 25: 180-229.

Bouchet P., Kantor Yu. I., Sysoev A. \& Puillandre N. 2011: A new operational classification of the Conoidea (Gastropoda). - Journal of Molluscan Studies 77: 273-308.

http://dx.doi.org/10.1093/mollus/eyr017

Brocchi G. 1814: Conchiologia fossile Subapennina, 2: 241-712, Stamperia Reale, Milano.

BRONN H. G. 1828: Verzeichniss der vom Heidelberger Mineralien-Komptoir herausgegebenen geognostisch-petrefaktologischen Sammlungen. - Zeitschrift für Mineralogie 2: 737-743.

Bruguik̀re M. 1792: Encyclopédie méthodique. Histoire naturelle des vers. 1. - Panckoucke, Paris, $757 \mathrm{pp}$.

Caze B., Saint Martin J.-P., Merle D. \& Saint Martin S. 2010: Intérêt des motifs colorés résiduels des coquilles de mollusques pour la valorisation des sites paléontologiques et des collections: l'exemple du Badénien de Roumanie. - In: Saint MARTin J.-P., Saint Martin S., OAie G., Seghedi A. \& Grigorescu D. (Coord.): Le patrimoine paléontologique. GeoEcoMar, Bucarest, pp. 27-38. 
Chira C. \& Voia I. 2001: Middle Miocene (Badenian) Conidae from Lăpugiu de Sus, Romania: systematical and palaeoecological data. - Studia Universitatis Babeş-Bolyai, Geologia 46(2): 151-160.

Chirli C. 1997: Malacofauna pliocenica Toscana. Vol. I, Superfamiglia Conoidea. - Firenze, 129 pp.

Csepreghy-Meznerics I. 1956: A szobi és letkési puhatestủ fauna. (Die Molluskenfauna von Szob und Letkés.) - Jahrbuch der Ungarischen Geologischen Anstalt 45(2): 363-442, (443-477).

Da Costa F. P. 1866: Gasteropodes dos depositos terciarios de Portugal. - Academia Real das Sciencias, Lisboa, $116 \mathrm{pp}$.

Davoli F. 1972: Conidae (Gastropoda). - In: Montanaro Gallitelli E. (ed.): Studi monografici sulla malacologia miocenica modenese, Parte I, I molluschi tortoniani di Montegibbio. - Palaeontographia Italica 68: 55-143.

Davoli F. 2003: I molluschi del Messiniano Inferiore di Borelli (Torino) 5. Conidae e Terebridae. - Bollettino del Museo Regionale di Scienze Naturali - Torino 20(2) (2002): 439-475.

Deshayes G. P. 1845: Histoire naturelle des animaux sans vertèbres, 11, Histoire des mollusques. Baillière, Paris, 665 pp.

Duş̧A A. 1967: Contribuții la studiul faunei Tortoniene de la Coşteiul de Sus-Nemeşeşti, regiunea Banat. - Studia Universitatis Babeş-Bolyai, Geologia-Geographia 1: 51-62.

DuşA A. 1969: Stratigrafia depozitelor Mezozoice şi Terţiare de la Căprioara - Coşteiu de Sus. - Bucureşti, $156 \mathrm{pp}$.

Ferrero Mortara E., Montefameglio l., Novelli M., Opesso G., Pavia G. \& Tampieri R. 1984: Catalogo dei tipi e degli esemplari figurati della collezione Bellardi e Sacco. Parte II. Museo Regionale di Scienze Naturali, Torino, 484 pp.

Fontannes F. 1879-1880: Les mollusques pliocènes de la Vallée du Rhône et du Roussillon. Vol. 1: Gastéropodes des formations marines et saumatres. - Lyon - Paris, 276 pp.

Glibert M. 1952: Gastropodes du Miocène moyen du Bassin de la Loire. - Koninklijk Belgisch Instituut voor Natuurwetenschappen, Verhandelingen 46: 241-450.

Gmelin J. F. 1791: Systema Naturae per Regna Tria Naturae. Tomus 1, pars 6. - Lipsiae, 3021-3910.

HaLAVÁTs J. 1876: Felső-Lapugy mediterrán faunája. [Miocene fauna of Felső-Lapugy.] - Földtani Közlöny 6(6-7): 229-240.

Halaváts J. 1881: Über die Verbreitung der in den Mediterran-Schichten von Ungarn vorkommenden Conus-Formen. - Földtani Közlöny 11(1-3): 56-58.

Hall C. A. 1964: Middle Miocene Conus (Class Gastropoda) from Piedmont, northern Italy. Bollettino della Società Paleontologica Italiana 3(2): 111-171.

Harzhauser M. \& Piller W. E. 2007: Benchmark data of a changing sea - Palaeogeography, palaeobiogeography and events in the Central Paratethys during the Miocene. - Palaeogeography, Palaeoclimatology, Palaeoecology 253: 8-31. http://dx.doi.org/10.1016/j.palaeo.2007.03.031

Hauer F. \& Stache G. 1863: Geologie Siebenbürgens. - Braumüller, Wien, 636 pp.

HendRICKs J. 2015: Glowing Seashells: Diversity of Fossilized Coloration Patterns on Coral ReefAssociated Cone Snail (Gastropoda: Conidae) Shells from the Neogene of the Dominican Republic. - PLoS ONE 10(4): e0120924. http://dx.doi.org/10.1371/journal.pone.0120924

Hoernes R. 1878: Ueber das Vorkommen des Genus Conus in den marinen Neogen-Ablagerungen der öster.-ungar. Monarchie. - Verhandlungen der Kaiserlich-Königlichen Geologischen Reichsanstalt 9: 191-196.

Hoernes R. \& Auinger M. 1879: Die Gasteropoden der Meeres-Ablagerungen der ersten und zweiten Miocänen Mediterran-Stufe in der Österreichisch-Ungarischen Monarchie. I. Conus. - Abhandlungen der Kaiserlich-Königlichen Geologischen Reichsanstalt 12(1): 1-52.

Hörnes M. 1853: Über die Tertiärversteinerungen von einem neuem Fundorte nächst dem Dorfe Nemesey im Banate. - Jahrbuch der Kaiserlich-Königlichen Geologischen Reichsanstalt 4(1): $192-193$. 
Hörnes M. 1856: Die fossilen Mollusken des tertiær-beckens von Wien. - Abhandlungen der Kaiserlich-Königlichen Geologischen Reichsanstalt 3: 1-736.

Janssen A. W., Janssen R., Tracey S., Vaessen L. \& Voort J. 2014a: History of a marine, Cainozoic gastropod taxon, Conus antidiluvianus Bruguière, 1792 and its nomenclatural implications. - Cainozoic Research 14(1): 73-90.

Janssen A. W., Janssen R., Tracey S., Vaessen L. \& Voort J. 2014b: Conus antidiluvianus Bruguière, 1792 (Mollusca, Gastropoda, Conidae): proposed conservation of prevailing usage of specific name by setting aside the unidentifiable lectotype and replacing it with a neotype. - Bulletin of Zoological Nomenclature 71(4): 223-229.

Косн A. 1889: Uj paläontologiai adatok Erdély ifjabb harmadkori képződményeiből. (Neue palaeontologische Daten aus den jüngeren Tértiárbildungen Siebenbürgens.) - Orvos-természettudományi Értesitó 14(2): 140-152, (176-181).

Косн A. 1898: Újabb megfigyelések és gyüjtés Felső-Lapugyon. (Neuere Beobachtungen und Aufsammlung in Felső-Lapugy.) - Földtani Közlöny 28(7-9): 209-226, (265-277).

Косн A. 1900: Die Tertiärbildungen des Beckens der Siebenbürgischen Landestheile. II. Neogene Abtheilung. - Budapest, $370 \mathrm{pp}$.

Koнn A. J. 2014: Conus of the Southeastern United States and Caribbean. - Princeton University Press, Princeton, $457 \mathrm{pp}$.

Kojumdgieva E. 1960: Le Tortonien du type viennois. - In: Kojumdgieva E. \& Strachimirov B.: Les fossiles de Bulgarie, VII, Tortonien, pp. 1-246, Academie des Sciences de Bulgarie, Sofia, $317 \mathrm{pp}$.

Kovács Z. \& Vicián Z. 2014: Badenian (Middle Miocene) Conoidean (Neogastropoda) fauna from Letkés (N Hungary). - Fragmenta Palaeontologica Hungarica 30(2013): 53-100.

Landau B. M., Harzhauser M., İslamoĞLu Y. \& Silva C. M. 2013: Systematics and palaeobiogeography of the gastropods of the middle Miocene (Serravallian) Karaman Basin, Turkey. - Cainozoic Research 11-13: 1-584.

Lóczy L. 1882: Geologische Notizen aus dem nördlichen Theile des Krassóer Comitates. - Földtani Közlöny 12(5-6): 119-138.

Michelotti G. 1847: Déscription des fossiles des terrains Miocènes de l'Italie septentrionale. - Natuurkundige Verhandelingen van de Hollandsche Maatschappij der Wetenschappen te Haarlem, 1-408.

MoIsescu G. 1955: Date noi asupra faunei de molluşte şi brachiopode tortoniene de la Lăpugiul superior. - Revista Universitatii „C. J. Parhon” şi a Politechnicii Bucureşti 8: 255-286.

MuÑiz R. 1999: El género Conus L., 1758 (Gastropoda, Neogastropoda) Plioceno de Estepona (Málaga, España). - Iberus 17(1): 31-90.

Nawrot R., Zuschin M., Harzhauser M., Kroh A. \& Mandic O. 2015: Local diversity hot spots in the Middle Miocene of the Central Paratethys: influence of environment and sampling. - Geophysical Research Abstracts 17: EGU2015-9948.

Neugeboren J. L. 1846: Foraminiferen aus bem Tegel-Thon bei Felsö-Lapugy, unweit Dobra. Transsilvania 7(94): 433-434.

Neugeboren J. L. 1847: Über die Foraminiferen des Tegels von Felsö-Lapugy. - Berichte über die Mittheilungen von Freunden der Naturwissenschaften in Wien 2(10): 163-164.

Neugeboren J. L. 1852: Notiz über das erst neuerlich entdeckte Lager tertiärer Conchylien bei dem Dorfe Nemesey im Banate ganz nahe der siebenbürgischen Gränze. - Verhandlungen und Mittheilungen des siebenbürgischen Vereins für Naturwissenschaften zu Hermannstadt 3(2): 155-159.

Neugeboren J. L. 1853: Beiträge zur Kenntniss der Tertiär-Mollusken aus dem Tegelgebilde von Ober-Lapugy, I. Familie der Involuta. - Verhandlungen und Mittheilungen des siebenbürgischen Vereins für Naturwissenschaften zu Hermannstadt 4(7): 129-136. 
Neugeboren J. L. 1854: Bericht über einen neuen Fundort tertiärer Conchylien bei dem Dorfe Kostej im Banate nächst der siebenb. Gränze. - Verhandlungen und Mittheilungen des siebenbürgischen Vereins für Naturwissenschaften zu Hermannstadt 5(9): 148-152.

Nicorici E. \& Sagatovici A. 1973: Étude de la faune du Badénien supérieur de Minisul de Sus (Bassin de Zarand). - Anuarul Institutului Geologic 40: 111-194.

Niţulescu O. 1931: Beiträge zur Geologischen Kenntnis der Gegend um Lăpugiul-de-Sus (Hunedoara, Siebenbürgen). - Revista Muzeului Geologic-Mineralogic al Universitații din Cluj 4(1) (1930): 27-46. (in Romanian)

Orbigny A. D' 1852: Podrome de Paléontologie stratigraphique universelle des Animaux Mollusques et Rayonnés, 3. - Masson, Paris, 190 pp.

Pacaud J.-M. 2003: First fossil records of the Recent Ovulid genus Pseudocypraea Schilder, 1927 (Mollusca, Gastropoda) with description of a new species. - Geodiversitas 25(3): 451-462.

Paganelli G. 2014: Fossil Conus from Italian Piacenzian Pliocene. - The Cone Collector 24: 29-39.

PAPP C. 1976: Biostratigrafia Neogenului din regiunea Läpugiu de Jos - Lăpugiu de Sus - Crivina (jud. Hunedoara). - Unpublished MSc thesis, Universitatea Babeş-Bolyai, Cluj-Napoca, pp. 154.

Petrescu I., Mészáros N., Chira C. \& Filipescu S. 1990: Lower Badenian paleoclimate at Lăpugiu de Sus (Hunedoara County), on account of paleontological investigations. - Studia Universitatis Babeş-Bolyai, Geologia 35(2): 13-22.

Popa M. V., Duma A., SĂPlăCAN A. 2015: Badenian gastropods from the collections of the Mureş County Museum. - Analele Stiintifice ale Universitatii "Al. I. Cuza" din Iasi Seria Geologie 60(2) (2014): 5-30.

SAcco F. 1893: I molluschi dei terreni terziarii del Piemonte e della Liguria, Parte 13. - Memorie della Reale Accademia delle scienze di Torino, ser. 2, 44, Fasc. 1 (Conidae): 1-54, Fasc. 2 (Conidae e Conorbidae): 55-143.

STraUSz L. 1966: Die miozän-mediterranen Gastropoden Ungarns. - Akadémiai Kiadó, Budapest, 692 pp.

STUR D. 1863: Bericht über die geologische Uebersichtsaufnahme des südwestlichen Siebenbürgen im Sommer 1860. - Jahrbuch der Kaiserlich-Königlichen Geologischen Reichsanstalt 16: 33-120.

TĂmAș A., TĂMAș D. M. \& Popa M. V. 2013: Badenian small gastropods from Lăpugiu de Sus (Făget Basin, Romania). Rissoidae family. - Acta Palaeontologica Romaniae 9(1): 57-66.

Tucker J. K. \& Tenorio M. J. 2009: Systematic Classification of Recent and Fossil Conoidean Gastropods. - ConchBooks, Hackenheim, 296 pp.

VAessen L. 2010: De Conus soorten uit het Langhian van het Loirebekken. - Afzettingen WTKG 31(1):5-15.

ZILCH A. 1934: Zur Fauna des Mittel-Miocäns von Kostej (Banat); Typus Bestimmung und Tafeln zu O. Boettger's Bearbeitungen. - Senckenbergiana 16: 193-302. 